\title{
Harmonic morphisms with 1-dimensional fibres on 4-dimensional Einstein manifolds
}

\author{
RADU PANTILIE ${ }^{1}$
}

\begin{abstract}
Harmonic morphisms are smooth maps between Riemannian manifolds which preserve Laplace's equation. They are characterised as harmonic maps which are horizontally weakly conformal [14, 20]. R.L. Bryant [7] proved that there are precisely two types of harmonic morphisms with one-dimensional fibres which can be defined on a constant curvature space of dimension at least four. Here we prove that, on an Einstein four-manifold, there are precisely three types of harmonic morphisms with one-dimensional fibres, the third type being new.

We have the following applications: let $\left(M^{4}, g\right)$ and $\left(N^{3}, h\right)$ be complete Einstein manifolds; then we list all the harmonic morphisms from $\left(M^{4}, g\right)$ onto $\left(N^{3}, h\right)$ when:

- $M^{4}$ and $N^{3}$ are simply-connected (Theorem 4.1, Theorem 4.8);

- $M^{4}$ is compact (Theorem 4.11).
\end{abstract}

\section{Introduction.}

It is useful to place the study of harmonic morphisms into the context of conformal foliations. The idea is due to J.C. Wood [34] (also, see [4] for a significant application of it). Say that a foliation produces harmonic morphisms if its leaves can be locally defined as fibres of harmonic morphisms. Then harmonic morphisms can be described and classified in terms of the geometrical properties of the foliations formed by their fibres. For example, on a Riemannian manifold of dimension at least four and with constant curvature there are just two types of one-dimensional foliations which produce harmonic morphisms (a result essentially due to R.L. Bryant [7]):

(i) Riemannian foliations locally generated by Killing fields and

\footnotetext{
${ }^{1}$ The author gratefully acknowledges the support of the O.R.S. Scheme Awards, the School of Mathematics of the University of Leeds, the Tetley and Lupton Scholarships and the Edward Boyle Bursary.
} 
(ii) homothetic foliations with geodesic leaves and integrable orthogonal complement. The type (i) is due to R.L. Bryant whilst type (ii) is due to P. Baird and J. Eells [3] (cf. [34]).

In [29] it is proved that the alternative (i) or (ii) still holds for onedimensional foliations which produce harmonic morphisms and have integrable orthogonal complement on Einstein manifolds of dimension at least four. One of the consequences of the main result of Section 1 below is that the integrability assumption cannot be removed, at least in dimension four. More precisely, in Theorem 1.8 we prove that on a four-dimensional Einstein manifold any one-dimensional foliation which produces harmonic morphisms is of one of the types (i), (ii) or (iii) where (i) and (ii) are as above and (iii) is as follows: $\left(M^{4}, g\right)$ is Ricci-flat and, up to homotheties, any harmonic morphism $\varphi:\left(U,\left.g\right|_{U}\right) \rightarrow\left(N^{3}, h\right)$, with dilation $\lambda$, produced by $\mathcal{V}$ such that $\left.\mathcal{V}\right|_{U}$ and $N^{3}$ are orientable is (locally) described by:

(a) $\left(N^{3}, h\right)$ has constant sectional curvature equal to one;

(b) $\frac{1}{2} \mathrm{~d}\left(\lambda^{-2}\right)$ is a (flat) principal connection for $\mathcal{V}$ with respect to suitably chosen $V \in \Gamma(\mathcal{V})$ such that $g(V, V)=\lambda^{2}$;

(c) the local connection form $A$ of $\mathcal{H}$ with respect to $\frac{1}{2} \mathrm{~d}\left(\lambda^{-2}\right)$ satisfies the equation $\mathrm{d} A+2 * A=0$ on $\left(N^{3}, h\right)$ where $*$ is the Hodge star-operator of $\left(N^{3}, h\right)$ with respect to some orientation of $N^{3}$.

The construction of the metric $g$ in type (iii) above appears to be in the spirit of work of P.E. Jones and K.P. Tod (see [9]).

Examples of harmonic morphisms of type (iii) are given in Section 2. There we prove that these are always submersive (Proposition 2.1). Also, we show that any surjective harmonic morphism of type (iii) with connected fibres and complete codomain is, up to homotheties and Riemannian coverings, the restriction of the radial projection $\left(\mathbb{R}^{4} \backslash\{0\}, g_{a}\right) \rightarrow S^{3}$ where $g_{a}$ is the Eguchi-Hanson II metric [12] ( $g_{0}$ is induced by the canonical metric on $\mathbb{R}^{4}$ and note that it is well-known that $\varphi_{0}$ is a harmonic morphism of type (ii)). In particular, there exists no surjective harmonic morphism of type (iii) with connected fibres whose domain and codomain are both complete.

A first set of applications of Theorem 1.8 is given in Section 3. For example we have: if $\varphi:\left(M^{4}, g\right) \rightarrow\left(N^{3}, h\right)$ is a nonconstant harmonic morphism between compact Einstein manifolds of dimension four and three, respectively, then $\left(M^{4}, g\right)$ and $\left(N^{3}, h\right)$ are flat and, up to homotheties and Riemannian coverings, $\varphi$ is the canonical projection between flat tori $T^{4} \rightarrow$ 
$T^{3}$. This is proved in Theorem 3.8 for submersive harmonic morphisms and Theorem 4.11, in general.

In Section 4 we study surjective harmonic morphisms $\varphi:\left(M^{4}, g\right) \rightarrow$ $\left(N^{3}, h\right)$ (the necessity of surjectivity was pointed out by J.C. Wood) between complete Einstein manifolds of dimension four and three, respectively. If $M^{4}$ and $N^{3}$ are simply-connected we prove the following:

- If $\varphi$ is submersive then, up to homotheties, it is one of the following projections $\mathbb{R}^{4} \rightarrow \mathbb{R}^{3}, H^{4} \rightarrow \mathbb{R}^{3}, H^{4} \rightarrow H^{3}$ induced by the following canonical warped-product decompositions $\mathbb{R}^{4}=\mathbb{R}^{1} \times \mathbb{R}^{3}, H^{4}=H^{1} \times_{r}$ $\mathbb{R}^{3}, H^{4}=H^{1} \times_{s} H^{3}$ where $H^{k}$ is the hyperbolic space of dimension $k$ (Theorem 4.1).

- If $\varphi$ has exactly one critical point then there exists $a \geq 0$ such that, up to homotheties, $\varphi:\left(\mathbb{R}^{4}, g_{a}\right) \rightarrow\left(\mathbb{R}^{3}, h_{0}\right)$ is the Hopf polynomial with $g_{a}$ the Hawking Taub-NUT metric $(a>0)$ and $g_{0}, h_{0}$ the canonical metrics on $\mathbb{R}^{4}, \mathbb{R}^{3}$, respectively (Theorem 4.10 ). Moreover, we show how the Hopf polynomial in the last mentioned result can be generalised such that to allow any number of critical points (Theorem 4.8).

In the Appendix we prove a result needed in the proof of Theorem 4.1; there we also give constructions of harmonic morphisms of type (i) or (ii) defined on Einstein manifolds not of constant curvature and which have integrable horizontal distribution - thus answering to a question raised by S. Gudmundsson.

I am deeply indebted to J.C. Wood for thoughtful guidance.

\section{Foliations of dimension one which produce harmonic morphisms on four-dimensional Einstein manifolds.}

Foliations whose leaves are locally fibres of (submersive) harmonic morphisms were introduced in [34]. We introduced the following terminology in [29].

Definition 1.1. Let $(M, g)$ be a (connected) Riemannian manifold and let $\mathcal{V}$ be (the tangent bundle of) a foliation on it.

We say that $\mathcal{V}$ produces harmonic morphisms on $(M, g)$ if each point of $M$ has an open neighbourhood $U$ which is the domain of a submersive harmonic morphism $\varphi:\left(U,\left.g\right|_{U}\right) \rightarrow(N, h)$ whose fibres are open subsets of the leaves of $\mathcal{V}$. We call $\varphi$ a harmonic morphism produced by $\mathcal{V}$. 
Since any harmonic morphism is horizontally weakly conformal, any foliation which produces harmonic morphisms is a conformal foliation [34]. We recall the following definition [29].

Definition 1.2. Let $\mathcal{V}$ be a distribution on the Riemannian manifold $(M, g)$. We shall say that $\mathcal{V}$ is homothetic if it is conformal and the mean curvature form of its orthogonal complement is closed.

Homothetic foliations are characterised by the property that their leaves are locally fibres of horizontally homothetic submersions, and that a foliation of codimension not equal to two which has minimal leaves produces harmonic morphisms if and only if it is a homothetic foliation [29].

To state the main result of this section we also need a definition which is a trivial generalization to foliations of the well-known concept of principal connection on a principal bundle. For simplicity, we give this definition just for one-dimensional foliations.

Definition 1.3. Let $\mathcal{V}$ be a one-dimensional foliation and let $V \in \Gamma(\mathcal{V})$ be a nowhere zero vector field tangent to $\mathcal{V}$.

A principal connection for $\mathcal{V}$ (with respect to $V$ ) is a complementary distribution $\mathcal{H} \subseteq T M, \mathcal{H} \oplus \mathcal{V}=T M$ such that $V$ is an infinitesimal automorphism of $\mathcal{H}$ (i.e., $\mathcal{H}$ is invariant under the local flow of $V$ ).

The connection form $\theta$ of $\mathcal{H}$ is the 'vertical' dual of $V$ (i.e., $\theta(V)=1$ and $\left.\theta\right|_{\mathcal{H}}=0$ ) and the curvature form of $\mathcal{H}$ is $\Omega=\mathrm{d} \theta$. Note that $\Omega$ is basic and it can be interpreted as the integrability tensor of $\mathcal{H}$ (indeed $\Omega(X, Y) V=-\mathcal{V}([X, Y])$ for any horizontal vector fields $X$ and $Y)$.

It is obvious that a one form $\theta$ defines a principal connection for (the one-dimensional foliation) $\mathcal{V}$ with respect to $V$ if and only if $\theta(V)=1$ and $\mathcal{L}_{V} \theta=0$.

Example 1.4. Let $\mathcal{V}$ be an orientable one-dimensional geodesic foliation on $(M, g)$. Then $\mathcal{H}\left(=\mathcal{V}^{\perp}\right)$ is a principal connection for $\mathcal{V}$ with respect to $U \in \Gamma(\mathcal{V})$ where $g(U, U)=1$. The connection form is $U^{b}$.

An orientable one-dimensional foliation $\mathcal{V}$ on $M$ admits a principal connection if and only if it is geodesible (i.e., there exists a Riemannian metric $h$ on $M$ such that the leaves of $\mathcal{V}$ are geodesics on $(M, h)$ ). Indeed, given the principal connection $\mathcal{H}$ (with respect to some $V \in \Gamma(\mathcal{V})$ ) if we choose any metric $h$ such that $h(V, V)=1$ and $h(V, X)=0$ for $X \in \mathcal{H}$ then the leaves of $\mathcal{V}$ are geodesics of $(M, h)$. Also the set of principal connections of 
$\mathcal{V}$ (if nonempty) with respect to a given nowhere zero vector field $V \in \Gamma(\mathcal{V})$ is an affine space over the linear space of basic one-forms: if $\theta_{j}, j=1,2$ are connection forms then $\theta_{1}-\theta_{2}$ is locally the pull back by $\varphi: U \rightarrow N$ of a one-form $A \in \Gamma\left(T^{*} N\right)$ where $U$ is an open subset of $M$ and the fibres of $\varphi$ are open subsets of leaves of $\mathcal{V}$. Fix $V \in \Gamma(\mathcal{V})$. Then in a neighbourhood of each point of $N$ a local section $s$ of $\varphi$ can be found which, in a neighbourhood of its image, defines a principal connection $\theta_{s}$ which is flat (i.e., $\mathrm{d} \theta_{s}=0$ ). If $\theta$ defines a principal connection then the one-form $A$ such that $\theta=\theta_{s}+\varphi^{*}(A)$ is the local connection form of $\theta$ with respect to $s$. Because $\mathcal{V}$ is one-dimensional we can define the local connection form of a principal connection with respect to a (local) flat principal connection by using any parallel section of the flat connection. Also note that the existence of a global flat principal connection impose severe restrictions to the topology of the foliation and of the manifold. For example, as is well known, if the leaves of $\mathcal{V}$ are the fibres of a principal bundle $\xi=\left(M, N, S^{1}\right)$ over the simply-conected $N$ and $\xi$ admits a flat principal connection then $\xi$ is trivial and, in particular, $M$ and $N \times S^{1}$ are diffeomorphic.

The orthogonal complement of a one-dimensional foliation which produces harmonic morphisms is a principal connection of it. To show this we first recall the following [29]:

\section{Definition 1.5.}

1) Let $\mathcal{V}$ be a conformal foliation on the Riemannian manifold $(M, g)$. A smooth positive function $\lambda: U \rightarrow \mathbb{R}$ on an open subset $U$ of $M$ will be called a local dilation of $\mathcal{V}$ if $\left.\mathcal{V}\right|_{U}$ is a Riemannian foliation on $\left(U,\left.\lambda^{2} g\right|_{U}\right)$. If $U=M$ then $\lambda$ is called a (global) dilation of $\mathcal{V}$.

2) Let $\mathcal{V}$ be a foliation which produces harmonic morphisms on the Riemannian manifold $(M, g)$. Let $\lambda$ be a local dilation of $\mathcal{V}$ which restricts to give dilations of harmonic morphisms produced by $\mathcal{V}$. Then $\rho=\lambda^{2-n}$ is called a local density of $\mathcal{V}$. If $\lambda$ is globally defined on $M$ then $\rho$ is called a (global) density.

The terminology of Definition 1.5(2) is motivated by the following fact.

Remark 1.6. Let $\mathcal{V}$ be a foliation which produces harmonic morphisms on $(M, g)$. Let $\omega$ be a local volume form for $\mathcal{V}$ and $\mathcal{H}=\mathcal{V}^{\perp}$. A positive smooth function $\rho$ is a local density for $\mathcal{V}$ if and only if $\rho^{\frac{1}{2-n}}$ is a local dilation of $\mathcal{V}$ and $\rho \omega$ is invariant under the parallel displacement determined by $\mathcal{H}$ [29]. 
Proposition 1.7. Let $\mathcal{V}$ be a one-dimensional foliation which produces harmonic morphisms on $\left(M^{n+1}, g\right)$ where $\operatorname{dim} M=n+1$. Let $\rho=e^{(2-n) \sigma}$ be a local density of $\mathcal{V}$. Supposing that $\mathcal{V}$ restricted to the domain of $\sigma$ is orientable let $V \in \Gamma(\mathcal{V})$ be such that $g(V, V)=e^{(2 n-4) \sigma}\left(=\rho^{-2}\right)$.

Then the horizontal distribution $\mathcal{H}\left(=\mathcal{V}^{\perp}\right)$ is a principal connection for $\mathcal{V}$ with respect to $V$.

Proof. From [29, Lemma 2.1] (or Remark 1.6) it follows that $[V, X]=0$ for any basic vector field $X \in \Gamma(\mathcal{H})$.

We now state the main result of this section.

Theorem 1.8. Let $\left(M^{4}, g\right)$ be an Einstein manifold of dimension four and $\mathcal{V}$ a one-dimensional foliation which produces harmonic morphisms on $\left(M^{4}, g\right)$.

Then, one of the following assertions holds:

(i) $\mathcal{V}$ is Riemannian and locally generated by Killing fields;

(ii) $\mathcal{V}$ is a homothetic foliation by geodesics with integrable orthogonal complement;

(iii) $\left(M^{4}, g\right)$ is Ricci-flat and, up to homotheties, any harmonic morphism $\varphi:\left(U,\left.g\right|_{U}\right) \rightarrow\left(N^{3}, h\right)$, with dilation $\lambda$, produced by $\mathcal{V}$ such that $\left.\mathcal{V}\right|_{U}$ and $N^{3}$ are orientable is (locally) described as follows:

(a) $\left(N^{3}, h\right)$ has constant sectional curvature $k^{N}=1$,

(b) $\frac{1}{2} \mathrm{~d}\left(\lambda^{-2}\right)$ is a (flat) principal connection for $\mathcal{V}$ with respect to suitably chosen $V \in \Gamma(\mathcal{V})$ such that $g(V, V)=\lambda^{2}$,

(c) the local connection form $A$ of $\mathcal{H}$ with respect to $\frac{1}{2} \mathrm{~d}\left(\lambda^{-2}\right)$ satisfies the equation $\mathrm{d} A+2 * A=0$ on $\left(N^{3}, h\right)$ where $*$ is the Hodge staroperator of $\left(N^{3}, h\right)$ with respect to some orientation of $N^{3}$.

Moreover, only (i) and (ii) or (ii) and (iii) can occur simultaneously, in which case $\left(M^{4}, g\right)$ must be Ricci-flat.

From Theorem 1.8 we obtain the following.

Corollary 1.9. Let $\left(M^{4}, g\right)$ be an orientable Einstein manifold of dimension four, and $\left(N^{3}, h\right)$ an orientable Riemannian manifold of dimension three. 
Let $\varphi:\left(M^{4}, g\right) \rightarrow\left(N^{3}, h\right)$ be a submersive harmonic morphism; denote its dilation by $\lambda$ and let $V \in \Gamma(\mathcal{V})$ be such that $g(V, V)=\lambda^{2}$.

Then, one of the following assertions (i), (ii), (iii) holds:

(i) $V$ is a Killing field;

(ii) $\varphi$ is horizontally homothetic and has geodesic fibres orthogonal to an umbilical foliation by hypersurfaces;

(iii) (a) $\left(M^{4}, g\right)$ is Ricci-flat and $\left(N^{3}, h\right)$ has constant sectional curvature $k^{N}=\frac{c^{2}}{4}(c>0)$,

(b) $\frac{1}{c} \mathrm{~d}\left(\lambda^{-2}\right)$ is a (flat) principal connection for $\operatorname{ker} \varphi_{*}$ with respect to $V$,

(c) any local connection form $A$ of $\left(\operatorname{ker} \varphi_{*}\right)^{\perp}$ with respect to $\frac{1}{c} \mathrm{~d}\left(\lambda^{-2}\right)$ satisfies $\mathrm{d} A+c * A=0$ on $\left(N^{3}, h\right)$ where $*$ is the Hodge staroperator of $\left(N^{3}, h\right)$ defined by some orientation of $N^{3}$.

\section{Remark 1.10.}

1) If $M^{4}$ is not orientable then we can replace $\left(M^{4}, g\right)$ by a Riemannian double covering $\left(\widetilde{M^{4}}, \widetilde{g}\right)$ such that $\widetilde{M^{4}}$ is orientable. Then we replace $\varphi$ by $\widetilde{\varphi}=\varphi \circ \xi$ where $\xi:\left(\widetilde{M^{4}}, \widetilde{g}\right) \rightarrow\left(M^{4}, g\right)$ is the projection of the covering.

2) If $N^{3}$ is not orientable we can pull-back $\varphi$ to a Riemannian doublecovering $\left(\widetilde{N^{3}}, \widetilde{h}\right)$ of $\left(N^{3}, h\right)$ such that $\widetilde{N^{3}}$ is orientable.

Before proving Theorem 1.8 we need some further preparations.

Let $\mathcal{V}$ be a one-dimensional foliation which produces harmonic morphisms on a Riemannian manifold $\left(M^{4}, g\right), \operatorname{dim} M=4$ and let $\rho=e^{-\sigma}$ be a positive smooth function. We define the Riemannian metric $h$ on $M$ by

$$
h=e^{2 \sigma} g^{\mathcal{H}}+e^{-2 \sigma} g^{\mathcal{V}}
$$

where $g^{\mathcal{H}}$ and $g^{\mathcal{V}}$ are the horizontal and the vertical part of $g$, respectively. Then from [29, Proposition 1.8] (cf. [27], [28]) it follows that $\mathcal{V}$ produces harmonic on $(M, h)$ aswell. Furthermore if $\rho=e^{-\sigma}$ is a (local) density of $\mathcal{V}$ then $\mathcal{V}$ is Riemannian and has geodesic leaves with respect to $h$.

Assuming that $\mathcal{V}$ restricted to the domain $O$ of the local density $\rho$ is orientable let $V \in \Gamma\left(\left.\mathcal{V}\right|_{O}\right)$ be such that $g(V, V)=\rho^{-2}$. Then, by Proposition $1.7, \mathcal{H}$ is a principal connection for $\mathcal{V}$, with respect to $V$. We shall always denote by $\Omega$ the curvature form of $\mathcal{H}$. 
Next we relate the Ricci tensors ${ }^{M}$ Ricci and ${ }^{N}$ Ricci of $\left(M^{4}, g\right)$ and $\left(N^{3}, \bar{h}\right)$, respectively, where $\left(N^{3}, \bar{h}\right)$ is the codomain of a harmonic morphism produced by $\mathcal{V}$.

Lemma 1.11. Let $O$ be an open subset of $M^{4}$ and let $\varphi:\left(O,\left.g\right|_{O}\right) \rightarrow(N, \bar{h})$ be a harmonic morphism produced by $\mathcal{V}$. Then,

$$
\begin{aligned}
{ }^{M} \operatorname{Ricci}(X, Y)= & N_{\operatorname{Ricci}}\left(\varphi_{*} X, \varphi_{*} Y\right)-\frac{1}{2} e^{4 \sigma} h\left(i_{X} \Omega, i_{Y} \Omega\right) \\
& -e^{-2 \sigma} \Delta^{M} \sigma h(X, Y)-2 X(\sigma) Y(\sigma), \\
{ }^{M} \operatorname{Ricci}(X, V)= & \frac{1}{2} e^{4 \sigma}\left({ }^{h} \mathrm{~d}^{*} \Omega\right)(X)+2 e^{4 \sigma} \Omega\left(X, \operatorname{grad}_{h} \sigma\right) \\
& +2 X(V(\sigma))-2 X(\sigma) V(\sigma), \\
& \\
{ }^{M} \operatorname{Ricci}(V, V)= & e^{2 \sigma} \Delta^{M} \sigma+\frac{1}{4} e^{8 \sigma}|\Omega|_{h}^{2}+4 V(V(\sigma))-10 V(\sigma)^{2}
\end{aligned}
$$

for any horizontal vectors $X, Y$, where $e^{\sigma}$ is the dilation of $\varphi, \Delta^{M}$ is the Laplacian on $(M, g)$ and ${ }^{h} \mathrm{~d}^{*}$ denotes the codifferential on $(M, h)$.

Proof. These equations follow, respectively, from (5.3), (5.4) and (4.4) of [29].

The following proposition which will be used later on holds for manifolds of any dimension.

Proposition 1.12. Let $(M, g)$ be an Einstein manifold and $\mathcal{V}$ a onedimensional foliation of codimension not equal to two which produces harmonic morphisms on $(M, g)$.

Then the following assertions are equivalent:

(i) $\mathcal{V}$ has basic mean curvature form;

(ii) $\mathcal{V}$ is a homothetic foliation.

Proof. This follows from [29, Proposition 5.13].

Theorem 1.8 extends the result of [7] for the dimension considered.

We recall the following result of [29]. 
Theorem 1.13. Let $(M, g)$ be an Einstein manifold of dimension at least four endowed with a one-dimensional foliation $\mathcal{V}$. Suppose that either $\mathcal{V}$ has integrable orthogonal complement or $\mathcal{V}$ is homothetic.

Then $\mathcal{V}$ produces harmonic morphisms if and only if either

(i) $\mathcal{V}$ is Riemannian and locally generated by Killing fields or

(ii) $\mathcal{V}$ is homothetic, has geodesic leaves and integrable orthogonal complement.

Moreover, if both (i) and (ii) hold then $(M, g)$ is Ricci-flat.

Proof of Theorem 1.8. By passing to a two-fold covering, if necessary, we can suppose that $\mathcal{V}$ is oriented. Also, by passing to a regular covering, if necessary, we can suppose that $\mathcal{V}$ admits a global density $\rho=e^{-\sigma}[30]$. Thus there exists $V \in \Gamma(\mathcal{V})$ such that $g(V, V)=e^{2 \sigma}$.

If $\Omega=0$ then we are done by Theorem 1.13. So suppose that $\Omega \neq 0$. Then, since $\Omega$ is basic, we can choose a local orthonormal frame $\{X, Y, Z\}$ of $\mathcal{H}$ with respect to $h$, made up of basic fields and such that

$$
i_{Z} \Omega=0 \text { and } \Omega(X, Y) \neq 0 .
$$

Then it is easy to see that

$$
\begin{gathered}
h\left(i_{X} \Omega, i_{Y} \Omega\right)=h\left(i_{Y} \Omega, i_{Z} \Omega\right)=h\left(i_{Z} \Omega, i_{X} \Omega\right)=0, \\
h\left(i_{X} \Omega, i_{X} \Omega\right)=h\left(i_{Y} \Omega, i_{Y} \Omega\right)=\Omega(X, Y)^{2} .
\end{gathered}
$$

Now recall that $\left(M^{4}, g\right)$ is Einstein and thus ${ }^{M_{\text {Ricci }}}=c^{M} g$ for some real number $c^{M} \in \mathbb{R}$. Hence from (1.1) and (1.4) it follows that

$$
X(\sigma) Y(\sigma), Y(\sigma) Z(\sigma), Z(\sigma) X(\sigma)
$$

are basic functions. Also, because ${ }^{M_{R}} \operatorname{Ricci}(X, X)={ }^{M_{R}} \operatorname{Ricci}(Y, Y)$ from (1.1) and (1.5), it follows that $X(\sigma)^{2}-Y(\sigma)^{2}$ is a basic function. Hence $X(\sigma), Y(\sigma)$ are basic functions and, moreover, outside the set

$$
S=\left\{x \in M \mid X_{x}(\sigma)=Y_{x}(\sigma)=0\right\},
$$

we have that $Z(\sigma)$ is also basic. It follows that, at least, outside the interior of $S$, the foliation $\mathcal{V}$ is homothetic by Proposition 1.12. From Theorem 1.13 we obtain that the alternative (i) or (ii) of Theorem 1.8 holds locally at 
least on $M \backslash S$ (just locally because $M \backslash S$ might be disconnected). If also $Z(\sigma)=0$ on $S$ then we are done because then $\mathcal{V}$ is homothetic on $M$ and we can again apply Theorem 1.13.

From now on in this proof we shall work on $\stackrel{\circ}{S} \cap\left\{x \in M \mid Z_{x}(\sigma) \neq 0\right\}$. There we can write $[X, Y](\sigma)=X(Y(\sigma))-Y(X(\sigma))=0$ and hence

$$
\begin{aligned}
-\mathcal{V}[X, Y](\sigma) & =\mathcal{H}[X, Y](\sigma) \\
\Longleftrightarrow \Omega(X, Y) V(\sigma) & =h\left(\mathcal{H}[X, Y], \operatorname{grad}_{h} \sigma\right) .
\end{aligned}
$$

Since we are in the interior of $S$ the last equality is equivalent to

$$
\Omega(X, Y) V(\sigma)=Z(\sigma) h([X, Y], Z) .
$$

Next we compute $\left({ }^{h} \mathrm{~d}^{*} \Omega\right)(Z)$ (note that by (1.2) the other components of $\mathcal{H}^{*}\left({ }^{h} \mathrm{~d}^{*} \Omega\right)$ are zero):

$$
\begin{aligned}
\left({ }^{h} \mathrm{~d}^{*} \Omega\right)(Z)= & -\left(\stackrel{h}{\nabla}_{X} \Omega\right)(X, Z)-\left(\stackrel{h}{\nabla}_{Y} \Omega\right)(Y, Z) \\
& -\left(\stackrel{h}{\nabla}_{Z} \Omega\right)(Z, Z)-\left(\stackrel{h}{\nabla_{V}} \Omega\right)(V, Z) .
\end{aligned}
$$

Because $i_{Z} \Omega=0$ we have $\left(\stackrel{h}{\nabla}_{Z} \Omega\right)(Z, Z)=0$ and because $\Omega$ is basic and $\mathcal{V}$ is geodesic with respect to $h$ we have $\left(\stackrel{h}{V}_{V} \Omega\right)(V, Z)=0$. Also

$$
\begin{aligned}
\left(\stackrel{h}{\nabla}_{X} \Omega\right)(X, Z) & =X(\Omega(X, Z))-\Omega\left(\stackrel{h}{\nabla}_{X} X, Z\right)-\Omega\left(X, \stackrel{h}{\nabla}_{X} Z\right) \\
& =-\Omega\left(X,{ }^{\circ}{ }_{X} Z\right) .
\end{aligned}
$$

Since $h\left(\stackrel{h}{\nabla}_{X} Z, Y\right)=-h\left(Z, \stackrel{h}{\nabla}_{X} Y\right)$ we have

$$
\left(\stackrel{h}{\nabla}_{X} \Omega\right)(X, Z)=\Omega(X, Y) h\left(Z, \stackrel{h}{\nabla}_{X} Y\right) .
$$

Similarly $\left(\stackrel{h}{\nabla}{ }_{Y} \Omega\right)(Y, Z)=\Omega(Y, X) h\left(Z, \stackrel{h}{\nabla}_{Y} X\right)$ and we obtain that

$$
\begin{aligned}
\left({ }^{\mathrm{d}} \mathrm{d}^{*} \Omega\right)(Z) & =-\Omega(X, Y) h\left(Z, \stackrel{h}{\nabla}_{X} Y\right)-\Omega(Y, X) h\left(Z, \stackrel{h}{\nabla}_{Y} X\right) \\
& =-\Omega(X, Y)\left\{h\left(Z, \stackrel{h}{\nabla}_{X} Y\right)-h\left(Z, \stackrel{h}{\nabla}_{Y} X\right)\right\} .
\end{aligned}
$$

We have proved that

$$
\left({ }^{h} \mathrm{~d}^{*} \Omega\right)(Z)=-\Omega(X, Y) h(Z,[X, Y]) .
$$


Now by (1.2) (with $X$ replaced by $Z$ ) we have

$$
\frac{1}{2} e^{4 \sigma}\left({ }^{h}{ }^{*} \Omega\right)(Z)+2 Z(V(\sigma))-2 Z(\sigma) V(\sigma)=0 .
$$

From (1.7) and (1.8) it follows that on $\stackrel{\circ}{S} \cap\left\{x \in M \mid Z_{x}(\sigma) \neq 0\right\}$ we have

$$
-\frac{1}{2} e^{4 \sigma} \Omega(X, Y) h(Z,[X, Y])+2 Z(V(\sigma))-2 Z(\sigma) V(\sigma)=0
$$

which together with (1.6) gives

$$
-\frac{1}{2} e^{4 \sigma} \frac{V(\sigma)}{Z(\sigma)} \Omega(X, Y)^{2}+2 Z(V(\sigma))-2 Z(\sigma) V(\sigma)=0 .
$$

Because ${ }^{M} \operatorname{Ricci}(X, X)={ }^{M} \operatorname{Ricci}(Z, Z)$ from relation (1.1) we obtain that the function $-\frac{1}{2} e^{4 \sigma} h\left(i_{X} \Omega, i_{X} \Omega\right)+2 Z(\sigma)^{2}$ is basic, equivalently, $-\frac{1}{2} e^{4 \sigma} \Omega(X, Y)^{2}+2 Z(\sigma)^{2}$ is basic. This implies that

$$
e^{4 \sigma} V(\sigma) \Omega(X, Y)^{2}=2 V(Z(\sigma)) Z(\sigma) .
$$

From (1.9) and (1.10) it follows that

$$
V(Z(\sigma))=2 V(\sigma) Z(\sigma)
$$

which is equivalent to the fact that $e^{-2 \sigma} Z(\sigma)$ is basic. Hence $Z\left(V\left(e^{-2 \sigma}\right)\right)=$ $V\left(Z\left(e^{-2 \sigma}\right)\right)=0$. This implies that, if $V\left(e^{-2 \sigma}\right)$ is nonconstant, then its level hypersurfaces are horizontal and hence $\mathcal{H}$ is integrable; then the proof follows from Theorem 1.13. There remains to be considered the case when $V\left(e^{-2 \sigma}\right)$ is a constant, say $c \in \mathbb{R}$. By replacing, if necessary, $V$ with $-V$ we can assume that $c \geq 0$. Since $V\left(e^{-2 \sigma}\right)$ is basic, $V\left(V\left(e^{-2 \sigma}\right)\right)=0$, equivalently,

$$
V(V(\sigma))=2 V(\sigma)^{2}
$$

From (1.8) and (1.11) it follows that

$$
\left({ }^{h} \mathrm{~d}^{*} \Omega\right)(Z)=-4 e^{-4 \sigma} Z(\sigma) V(\sigma) .
$$

From (1.10) and (1.11) it follows that either

$$
\text { (a) } V(\sigma)=0 \quad \text { or } \quad(b) \Omega(X, Y)^{2}=4 e^{-4 \sigma} Z(\sigma)^{2}
$$

which, after replacing, if necessary, one of the vector fields $X, Y$ or $Z$ with its negative, is equivalent to

$$
\Omega(X, Y)=Z\left(e^{-2 \sigma}\right) .
$$


In case (a), $\mathcal{V}$ is Riemannian and the proof follows from Theorem 1.13. So suppose from now on that (1.14) holds.

By (1.11) the horizontal component of $\mathrm{d}\left(e^{-2 \sigma}\right)$ is basic and thus we can locally write

$$
\mathcal{H}^{*}\left(\mathrm{~d}\left(e^{-2 \sigma}\right)\right)=\varphi^{*}(B)
$$

where $\varphi$ is as in Lemma 1.11 and $B$ is a one-form on $N$. Hence $\varphi^{*}(\mathrm{~d} B)+$ $c \mathrm{~d} \theta=0$ where $\theta$ is, as before, the vertical dual of $V$. But $\Omega$ is also basic and hence $\Omega=\varphi^{*}(F)$ for some two-form $F$ on $N$. It follows that

$$
\mathrm{d} B=-c F \text {. }
$$

Because $\mathcal{V}$ is Riemannian and has geodesic leaves with respect to $h$ we have that $\varphi^{*}\left({ }^{h^{*}} F\right)=\mathcal{H}^{*}\left({ }^{h} \mathrm{~d}^{*} \Omega\right)$ where ${ }^{\bar{h}} \mathrm{~d}^{*}$ is the codifferential on $(N, \bar{h})$.

Now (1.13) can be written:

$$
\bar{h}_{\mathrm{d}} F=-c B .
$$

Also (1.14) can be written:

$$
F=* B \quad \text { (equivalently, } * F=B \text { ) }
$$

where $*$ is the Hodge star-operator (locally) induced on $(N, \bar{h})$ by the (local) orientation corresponding to $\left\{\varphi_{*} X, \varphi_{*} Y, \varphi_{*} Z\right\}$.

From (1.1) it easily follows that

$$
\begin{aligned}
& { }^{N} \operatorname{Ricci}\left(\varphi_{*} X, \varphi_{*} Y\right)={ }^{N} \operatorname{Ricci}\left(\varphi_{*} Y, \varphi_{*} Z\right)={ }^{N} \operatorname{Ricci}\left(\varphi_{*} Z, \varphi_{*} X\right)=0, \\
& { }^{N} \operatorname{Ricci}\left(\varphi_{*} X, \varphi_{*} X\right)={ }^{N} \operatorname{Ricci}\left(\varphi_{*} Y, \varphi_{*} Y\right) .
\end{aligned}
$$

Also by (1.1), ${ }^{N} \operatorname{Ricci}\left(\varphi_{*} X, \varphi_{*} X\right)={ }^{N} \operatorname{Ricci}\left(\varphi_{*} Z, \varphi_{*} Z\right)$ if and only if (1.14) holds after replacing, if necessary, one of the vector fields $X, Y$ or $Z$ with its negative. Thus $\left(N^{3}, \bar{h}\right)$ is Einstein and, because it is three-dimensional, it is of constant sectional curvature $k^{N}$. Then ${ }^{N}$ Ricci $=2 k^{N} \bar{h}$.

Now from (1.3) we obtain

$$
e^{2 \sigma} c^{M}=e^{2 \sigma} \Delta^{M} \sigma+\frac{1}{2} e^{8 \sigma} \Omega(X, Y)^{2}+4 V(V(\sigma))-10 V(\sigma)^{2}
$$

which together with (1.12) gives

$$
e^{-2 \sigma} c^{M}=e^{-2 \sigma} \Delta^{M} \sigma+\frac{1}{2} e^{4 \sigma} \Omega(X, Y)^{2}-2 e^{-4 \sigma} V(\sigma)^{2} .
$$


It is easy to see by using (1.14) that (1.19) implies

$$
\frac{1}{2} \Delta^{M}\left(e^{-2 \sigma}\right)+e^{-2 \sigma} c^{M}+c^{2}=0 .
$$

Also by (1.1) we have

$$
e^{-2 \sigma} c^{M}={ }^{N} \operatorname{Ricci}\left(\varphi_{*} X, \varphi_{*} X\right)-\frac{1}{2} e^{4 \sigma} \Omega(X, Y)^{2}-e^{-2 \sigma} \Delta^{M} \sigma .
$$

From formulae (1.19) and (1.21) it follows that

$$
\begin{aligned}
{ }^{N} \operatorname{Ricci}\left(\varphi_{*} X, \varphi_{*} X\right) & =e^{-2 \sigma} c^{M}+\frac{1}{2} e^{4 \sigma} \Omega(X, Y)^{2}+e^{-2 \sigma} \Delta^{M} \sigma \\
& =e^{-2 \sigma} c^{M}+\left(e^{-2 \sigma} c^{M}+2 e^{-4 \sigma} V(\sigma)^{2}\right)
\end{aligned}
$$

and thus

$$
{ }^{N} \operatorname{Ricci}\left(\varphi_{*} X, \varphi_{*} X\right)=2 e^{-2 \sigma} c^{M}+2 e^{-4 \sigma} V(\sigma)^{2} .
$$

Now recall that $(N, \bar{h})$ is of constant sectional curvature $k^{N}$ and thus (1.22) is equivalent to

$$
k^{N}=e^{-2 \sigma} c^{M}+e^{-4 \sigma} V(\sigma)^{2} .
$$

Now recall that $V\left(e^{-2 \sigma}\right)=c=$ constant and thus (1.23) reads

$$
k^{N}=e^{-2 \sigma} c^{M}+\frac{c^{2}}{4} .
$$

Now, if $\mathcal{V}$ is not Riemannian (i.e., $c>0$ ), then $e^{-2 \sigma}$ cannot be constant, and hence $c^{M}=0$ and $k^{N}=\frac{c^{2}}{4}>0$. Then, after a homothetic transformation, if necessary, we can suppose that $k^{N}=1$ and hence $c=2$. Then $\frac{1}{2} \mathrm{~d}\left(e^{-2 \sigma}\right)$ is a flat principal connection for $\mathcal{V}$ with respect to $V$. Put $A=-\frac{1}{2} B$. From equation (1.15) and the fact that $V\left(e^{-2 \sigma}\right)=2$ and it follows that

$$
\theta=\frac{1}{2} \mathrm{~d}\left(e^{-2 \sigma}\right)+\varphi^{*}(A)
$$

and hence $A$ is a local connection form of $\theta$ with respect to $\frac{1}{2} \mathrm{~d}\left(e^{-2 \sigma}\right)$. Also $\mathrm{d} A=F$ and this together with (1.18) implies that

$$
\mathrm{d} A+2 * A=0 .
$$


We have proved that, at each point of $M$, one of the situations (i), (ii) or (iii) occurs.

Now by (1.20) it is obvious that there cannot exist any point where both (i) and (iii) occur.

Suppose that in an open connected subset $O \subseteq M$ which is the domain of the harmonic morphism $\varphi:\left(O,\left.g\right|_{O}\right) \rightarrow(N, \bar{h})$ both situations (ii) and (iii) occurs. Then on the image (by $\varphi$ ) of the set where (iii) occurs from (1.25) it follows that

$$
\triangle A=4 A .
$$

But, on the image of the interior of the set where (ii) holds, the equation (1.26) is trivially satisfied. Thus (1.26) is satisfied on $N$; this is an analytic manifold because $(N, \bar{h})$ has constant curvature. By the regularity of solutions for elliptic operators (see [6, p. 467]) we have that $A$ is analytic and hence, if $O$ contains interior points of the set where (ii) occurs, we have that $A=0$ on $N$. Hence if the set where (iii) occurs is nonempty and also the interior of the set where (ii) occurs is nonempty then the set where (iii) occurs is contained by the set of points where (ii) occurs.

Since by Theorem 1.13 on each connected component of the complement of the set where (iii) occurs the alternative (i) or (ii) holds globally the theorem is proved.

\section{Remark 1.14.}

1) Note that if $A$ satifies (1.25) then $F=\mathrm{d} A$ satifies

$$
\bar{h}^{*} F+2 * F=0 .
$$

2) The codomain of a harmonic morphism of type (iii) of Theorem 1.8 always has constant positive sectional curvature. In the limit, when this tends to zero, we obtain a harmonic morphism of type (i). This follows from (1.24).

3) Harmonic morphisms of type (iii) are also of type (ii) if and only if $A=0$.

4) The result of Theorem 1.8 shows that, on an Einstein manifold of dimension four, the nonlinear system of partial differential equations whose solutions are harmonic morphisms with fibres of dimension one can be reduced to one of three types of systems of linear partial differential equations of the first order. For type (i) this is Killing's equation, 
and for type (iii) it is (1.25). Finally, the one-dimensional foliation $\mathcal{V}$ on $(M, g)$ is of type (ii) if and only if it is locally generated by vector fields $W \in \Gamma(\mathcal{V})$ which satisfies $4 \nabla W=\operatorname{div} W \operatorname{Id}_{T M}$ where $\nabla$ is the Levi-Civita connection of $(M, g)$ (see [30, Lemma 6.5]).

See [35] for other situations in which the nonlinear system of partial differential equatons whose solutions are harmonic morphisms can be reduced to a linear system of partial differential equations of the first order.

\section{The third type.}

We shall say that a harmonic morphism $\varphi:\left(M^{4}, g\right) \rightarrow\left(N^{3}, h\right)$ is of type (iii) ((i), (ii)) if its regular fibres form a foliation of type (iii) ((i), (ii)) of Theorem 1.8. In this section the harmonic morphisms of type (iii) will be the main object of study.

The first thing to be noticed about the harmonic morphisms of type (iii) is that they are always submersive.

Proposition 2.1. Let $\varphi:\left(M^{4}, g\right) \rightarrow\left(N^{3}, h\right)$ be a harmonic morphism of type (iii).

Then $\varphi$ is submersive.

Proof. By passing, if necessary, to a two-fold covering, we can suppose that the vertical distribution $\mathcal{V}$ (which is well-defined outside the set of critical points) is orientable. Then, as before, let $V \in \Gamma(\mathcal{V})$ be such that $g(V, V)=$ $\lambda^{2}$ where $\lambda$ is the dilation of $\varphi$. Since, up to a multiplicative constant, $\mathrm{d}\left(\lambda^{-2}\right)$ is a (flat) principal connection with respect to $V, V\left(\lambda^{-2}\right)$ is a nonzero constant. This implies that the connected components of any regular fibre of $\varphi$ are noncompact.

Suppose that $\varphi$ is not submersive and let $x_{0} \in M$ be a critical point of it. Recall that, by a result of P. Baird [2, Proposition 5.1], the set of critical points of $\varphi$ must be discrete. Then from the main result of [10] it follows that $\varphi$ is topologically locally equivalent at $x_{0}$ to the cone of the Hopf fibration $S^{3} \rightarrow S^{2}$. Hence, in a neighbourhood of $x_{0}$, the connected components of the regular fibres of $\varphi$ are diffeomorphic to $S^{1}$. But we have seen that all the regular fibres of $\varphi$ have noncompact connected components and hence $\varphi$ cannot have critical points.

Remark 2.2. Let $\varphi:\left(M^{4}, g\right) \rightarrow\left(N^{3}, h\right)$ be a harmonic morphism of type 
(iii) with connected fibres; denote its dilation by $\lambda$. Then $\mathcal{H}^{*}\left(\mathrm{~d}\left(\lambda^{-2}\right)\right)$ is a basic one-form and let $A \in \Gamma\left(T^{*} N\right)$ be such that $-\frac{1}{2} \mathcal{H}^{*}\left(\mathrm{~d}\left(\lambda^{-2}\right)\right)=\varphi^{*}(A)$. Because $\left(N^{3}, h\right)$ is of constant curvature it is an analytic manifold. But $A$ satisfies (1.26) and hence it is analytic. Using this fact it is easy to see that if $N^{3}$ is orientable then there exists an orientation of it such that $\mathrm{d} A+2 * A=0$ on $N^{3}$.

From the proof of Theorem 1.8 it follows that any harmonic morphism of type (iii) is locally determined by the local connection form $A$. This is also illustrated by the following example.

Example 2.3. Let $h$ be the canonical metric on the three-dimensional sphere $S^{3}$. Let $A=i^{*}\left(-x^{2} \mathrm{~d} x^{1}+x^{1} \mathrm{~d} x^{2}-x^{4} \mathrm{~d} x^{3}+x^{3} \mathrm{~d} x^{4}\right)$ where $i: S^{3} \hookrightarrow \mathbb{R}^{4}$ is the canonical inclusion.

Let $*$ be the Hodge star-operator on $\left(S^{3}, h\right)$ considered with the usual orientation of $S^{3}$. Then

$$
\mathrm{d} A-2 * A=0 .
$$

To show this, first note that $A$ is the canonical connection (form) on the Hopf bundle $\left(S^{3}, S^{2}, S^{1}\right)$. Also $|A|=1$ and thus it suffices to verify that $A \wedge \mathrm{d} A=2 v_{S^{3}}$ where $v_{S^{3}}$ is the usual volume form on $S^{3}$.

For $a \in \mathbb{R}$ let $g_{a}$ be the Riemannian metric on $\mathbb{R}^{4} \backslash\{0\}=(0, \infty) \times S^{3}$ defined by

$$
g_{a}=\rho^{2} h+\rho^{-2}(\rho \mathrm{d} \rho+a A)^{2} .
$$

Then for any $a \neq 0$ the canonical projection $\varphi_{a}:\left(\mathbb{R}^{4} \backslash\{0\}, g_{a}\right) \rightarrow\left(S^{3}, h\right)$ is a harmonic morphism of type (iii) whilst $g_{0}$ is the restriction to $\mathbb{R}^{4} \backslash\{0\}$ of the canonical metric on $\mathbb{R}^{4}$ and thus $\varphi_{0}: \mathbb{R}^{4} \backslash\{0\} \rightarrow S^{3}$ is the usual radial projection which is of type (ii).

Note that $\left(\mathbb{R}^{4} \backslash\{0\}, g_{a}\right)$ is the Eguchi-Hanson II metric [12] and thus is Ricci-flat and anti-self-dual.

Let $\psi_{a}=\pi \circ \varphi_{a}$ where $\pi: S^{3} \rightarrow S^{2}$ is the Hopf fibration. Then $\psi_{a}$ is a harmonic morphism with totally geodesic fibres. Any fibre of it is isometric with $\left(\mathbb{R}^{2} \backslash\{0\}, \gamma_{a}\right)$ where $\gamma_{a}$, in polar coordinates $(\rho, \theta)$, is given by

$$
\gamma_{a}=\rho^{2} \mathrm{~d} \theta^{2}+\rho^{-2}(\rho \mathrm{d} \rho+a \mathrm{~d} \theta)^{2} .
$$

It is easy to see that any point of $\mathbb{R}^{2} \backslash\{0\}$ is at finite distance from 0 with respect to $\gamma_{a}$. Hence $\left(\mathbb{R}^{2} \backslash\{0\}, \gamma_{a}\right)$ is not complete. Because the fibres of $\psi_{a}$ are closed and totally geodesic we obtain that $g_{a}$ is not complete for any $a \in \mathbb{R}$. 
We shall prove that the $\varphi_{a}$ of Example 2.3 are, essentially, the only surjective harmonic morphisms of type (iii) with connected fibres and complete simply-connected codomain. For this we need the following:

Proposition 2.4. Let $S^{3}(=\mathrm{Sp}(1))$ be the three-dimensional sphere considered with its canonical metric and orientation and let $*$ be the Hodge star-operator on it.

(i) The space of solutions of the equation

$$
\mathrm{d} A+2 * A=0, \quad A \in \Gamma\left(T^{*} S^{3}\right)
$$

is the space of left-invariant one-forms on $S^{3}$.

(ii) The space of solutions of the equation

$$
\mathrm{d} A-2 * A=0, \quad A \in \Gamma\left(T^{*} S^{3}\right)
$$

is the space of right-invariant one-forms on $S^{3}$.

Proof. (i) Let $S^{3} \times \mathrm{Sp}(1) \rightarrow S^{3}$ be the unique spin-structure on $S^{3}$ and let $S^{3} \times \mathbb{H} \rightarrow S^{3}$ be the spinor bundle induced by the action of the Clifford algebra $C l_{3}=\mathbb{H} \oplus \mathbb{H}$ on $\mathbb{H}$ given by $(x, y) \cdot q=x \cdot q$.

Consider the trivialization $T S^{3}=S^{3} \times \operatorname{Im} \mathbb{H}$ induced by the canonical left action of $S^{3}(=\mathrm{Sp}(1))$ on itself. Thus any one-form $A$ on $S^{3}$ can be viewed as a spinor field $A: S^{3} \rightarrow \operatorname{Im} \mathbb{H} \subseteq \mathbb{H}$ which is constant if and only if the corresponding one-form is left-invariant.

Consider the Dirac operator $D$ obtained by using the trivial flat connection on $S^{3} \times \mathbb{H} \rightarrow S^{3}$. Then it is easy to see that $A \in \Gamma\left(T^{*} S^{3}\right)$ satisfies (2.1) if and only if $D A=0$. Also a straightforward calculation gives $D^{2}=\Delta+2 D$ where $\Delta$ is the usual Laplacian acting on $\mathbb{H}$-valued functions on $S^{3}$. Thus any solution $A$ of (2.1) induces a harmonic $\mathbb{H}$-valued function on $S^{3}$ which must be constant if $A$ is globally defined on $S^{3}$.

(ii) Since the isometry $x \mapsto x^{-1}$ of $S^{3}$ reverses the orientation, it pulls back solutions of (2.1) to solutions of (2.2). Thus the proof of (ii) follows from (i).

Remark 2.5. There are other ways to describe the solutions of the equations (2.1) and (2.2). For example, since any orthogonal complex structure on $\mathbb{R}^{4}(=\mathbb{H})$ compatible with the canonical orientation can be described as 
left multiplication with imaginary quaternions of length one (see [8]) any solution of (2.2) is, up to a multiplicative constant, of the form

$$
A=i^{*}\left(\sum_{a, b} J_{b}^{a} x^{b} \mathrm{~d} x^{a}\right)
$$

where $J$ is any orthogonal complex structure which induce the canonical orientation on $\mathbb{R}^{4}$ (i.e., if $\left\{u_{1}, u_{2}\right\}$ is a complex basis of $\left(\mathbb{R}^{4}, J\right)$ then $\left\{u_{1}, J u_{1}, u_{2}, J u_{2}\right\}$ is positively oriented) and $i: S^{3} \hookrightarrow \mathbb{R}^{4}$ is the canonical inclusion. This can also be checked directly.

Also, any solution $A$ of (2.2) can be written $A=* i^{*}(F)$ where $F \in$ $\Lambda_{+}^{2}\left(\mathbb{R}^{4}\right)$ is a self-dual two-form.

In fact, by using these characterisations an alternative proof for Proposition 2.4 can be obtained. First, note that, for each one of the equations (2.1) and (2.2) we have a three dimensional space of solutions. Then, it is easy to see that if $A$ satisfies (2.1) or (2.2) then $A$ is coclosed and $\Delta A=4 A$ where $\Delta$ is the Hodge Laplacian on $S^{3}$. Thus $A$ is in the eigenspace corresponding to the first eigenvalue of $\Delta$ acting on coclosed one-forms of $S^{3}$ and it is well-known that this space is of dimension six (see [16, 7.2] or apply one of the results from [26, p. 148] and [21, Theorem 2.3]).

Proposition 2.6. Let $\varphi:\left(M^{4}, g\right) \rightarrow\left(N^{3}, h\right)$ be a surjective harmonic morphism of type (iii) such that $\left(N^{3}, h\right)$ is complete, simply-connected and $\varphi$ has connected fibres.

Then there exists a $\in \mathbb{R}$ such that, up to homotheties, $\varphi$ is a restriction of $\varphi_{a}:\left(\mathbb{R}^{4} \backslash\{0\}, g_{a}\right) \rightarrow\left(S^{3}, h\right)$ from Example 2.3.

Proof. Up to a homothety, we can identify $\left(N^{3}, h\right)$ with $S^{3}$ considered with its canonical metric and orientation. Let $\lambda$ be the dilation of $\varphi$. Then, by Proposition 2.4, there exists $a \in \mathbb{R}$ such that, up to an isometry of $S^{3}$, $-\frac{1}{2} \mathcal{H}^{*}\left(\mathrm{~d}\left(\lambda^{-2}\right)\right)=a \varphi^{*}(A)$ where $A \in \Gamma\left(T^{*} S^{3}\right)$ is as in Example 2.3. By Proposition 2.1, $\varphi$ is submersive and let $\mathcal{V}=\operatorname{ker} \varphi_{*}$. Because $\mathcal{V}$ is orientable we can find $V \in \Gamma(\mathcal{V})$ such that $g(V, V)=\lambda^{2}$.

Because $\varphi$ is of type (iii) we have that $V\left(\lambda^{-2}\right)$ is a nonzero constant. This implies that the restriction of $\lambda$ to any fibre of $\varphi$ is a diffeomorphism onto some open subinterval of $(0, \infty)$. Hence the map $\Phi: M^{4} \rightarrow S^{3} \times(0, \infty)$ defined by $\Phi(x)=\left(\varphi(x), \lambda(x)^{-1}\right), x \in M^{4}$, is a diffeomorphic embedding.

Then from the proof of Theorem 1.8 it follows that $\Phi:\left(M^{4}, g\right) \rightarrow$ $\left(S^{3} \times(0, \infty), g_{a}\right)$ is a local isometry and hence an isometric embedding. Also, it is obvious that $\varphi_{a} \circ \Phi=\varphi$. 
Corollary 2.7. Let $\varphi:\left(M^{4}, g\right) \rightarrow\left(N^{3}, h\right)$ be a surjective harmonic morphism of type (iii) with connected fibres such that $\left(N^{3}, h\right)$ is complete.

Then $\left(M^{4}, g\right)$ is not complete.

Proof. Up to homotheties, the universal covering of $\left(N^{3}, h\right)$ is $S^{3}$ with its canonical metric and orientation. Then, $\varphi$ can be pulled back via $S^{3} \rightarrow N^{3}$ to a harmonic morphism whose total space is complete if and only if $\left(M^{4}, g\right)$ is complete. Then we can define $\Phi$ as in the proof of Proposition 2.6. Then $\Phi$ is a local isometry and because $\left(\mathbb{R}^{4} \backslash\{0\}, g_{a}\right)$ is not complete $\left(M^{4}, g\right)$ is not complete.

\section{A few applications.}

From Theorem 1.8 and Corollary 2.7 we can exclude the third type in the complete case as follows:

Theorem 3.1. Let $\left(M^{4}, g\right)$ be a complete Einstein manifold of dimension four and let $\left(N^{3}, h\right)$ be a complete Riemannian manifold of dimension three.

Let $\varphi:\left(M^{4}, g\right) \rightarrow\left(N^{3}, h\right)$ be a surjective harmonic morphism with connected fibres.

Then, either:

(i) the regular fibres of $\varphi$ form a Riemannian foliation locally generated by Killing fields or

(ii) $\varphi$ is horizontally homothetic and has geodesic fibres orthogonal to an umbilical foliation by hypersurfaces.

Recall that for a horizontally weakly-conformal map the vertical distribution is well-defined outside the set of critical points. We analyse the behaviour of $\varphi$ at a critical point. The model is the cone of the Hopf fibration $S^{3} \rightarrow S^{2}$ which, as is well-known, can be written as a quadratic polynomial:

Definition 3.2. The Hopf polynomial is the harmonic morphism $\varphi: \mathbb{R}^{4} \rightarrow$ $\mathbb{R}^{3}$ defined by

$$
\varphi\left(z^{1}, z^{2}\right)=\left(\left|z^{1}\right|^{2}-\left|z^{2}\right|^{2}, 2 z^{1} \overline{z^{2}}\right)
$$

via the standard identifications $\mathbb{R}^{4}=\mathbb{C}^{2}, \mathbb{R}^{3}=\mathbb{R} \times \mathbb{C}$.

Note that the Hopf polynomial has an isolated critical point at the origin of $\mathbb{R}^{4}$. 
Corollary 3.3. Let $\left(M^{4}, g\right)$ be an Einstein manifold of dimension four.

Let $\varphi:\left(M^{4}, g\right) \rightarrow\left(N^{3}, h\right)$ be a nonconstant harmonic morphism which has critical points and orientable vertical distribution.

Then there exists a Killing field $V \in \Gamma(T M)$ tangent to the fibres of $\varphi$ which vanishes precisely at the critical points of $\varphi$. Moreover, in a neighbourhood of each critical point $\varphi$ is smoothly equivalent to the Hopf polynomial.

Proof. Because $\varphi$ has critical points and $\left(M^{4}, g\right)$ is Einstein, $\varphi$ must be of type (i). (By Proposition 2.1 it cannot be of type (iii) and from the main result of [15] it follows that $\varphi$ cannot be of type (ii).)

Let $\lambda$ be the dilation of $\varphi$ and let $\mathcal{V}$ be the vertical distribution of $\varphi$. Let $V \in \Gamma(\mathcal{V})$ be such that $g(V, V)=\lambda^{2}$.

Obviously, $V$ can be extended to a continuous vector field on $M$ whose zero set is equal to $C_{\varphi}$, the set of critical points of $\varphi$.

Then $V$ is a Killing field on $\left(M \backslash C_{\varphi},\left.g\right|_{M \backslash C_{\varphi}}\right)$ [7] (cf. [5], [29]). Hence it satisfies the equation

$$
\nabla^{*} \nabla V={ }^{M_{\text {Ricci }}(V)}
$$

(see, for example, [21, page 44]), here ${ }^{M}$ Ricci $\in \Gamma\left(T M \otimes T^{*} M\right)$ denotes the $(1,1)$ tensor field associated to the Ricci tensor of $\left(M^{4}, g\right)$. From the regularity of solutions for the elliptic operators it follows that $V$ is a smooth (in fact, analytic) vector field on $M$.

By Baird's result ([2, Proposition 5.1]) $\varphi$ has isolated critical points. Then in a neighbourhood of the critical point $x \in M$ the local flow of $V$ is equivalent, via the exponential map at $x$, with the flow $\left(\xi_{t}\right)$ on $T_{x} M$ given by the vector field with value $\nabla_{w} V$ at $w \in T_{x} M$. Recall that $(\nabla V)_{x}$ : $T_{x} M \rightarrow T_{x} M$ induces an orthogonal complex structure on $\left(T_{x} M, g_{x}\right)$ (see [21]). Hence $\left(\xi_{t}\right)$ induces an $S^{1}$ action on $T_{x} M$ which must be free outside zero since its quotient is equivalent to $\varphi$ in a neighbourhood of $x$. From this fact the last assertion of the proposition easily follows.

Remark 3.4. Since (3.1) holds in general, the conclusion of the above corollary holds for any harmonic morphism $\varphi:\left(M^{4}, g\right) \rightarrow\left(N^{3}, h\right)$ of type (i) and with orientable vertical distribution.

Corollary 3.5. Let $\left(M^{4}, g\right)$ be a complete Einstein manifold of dimension four and let $\left(N^{3}, h\right)$ be a complete Riemannian manifold of dimension three.

Let $\varphi:\left(M^{4}, g\right) \rightarrow\left(N^{3}, h\right)$ be a surjective submersive harmonic morphism with connected fibres and orientable vertical distribution; denote its dilation 
by $\lambda$. Let $W$ be a vertical vector field such that $g(W, W)=\lambda^{-2}$ and suppose that $W$ is complete.

Then $\varphi$ is of type (i) and there exists a globally defined nowhere zero Killing field tangent to the fibres of $\varphi$.

Proof. Suppose that (ii) of Theorem 3.1 occurs. Then, either directly or by using [29, Lemma 4.3], it can be shown that $\nabla W=\mu \operatorname{Id}_{T M}$ where $\mu$ is a smooth function on $M^{4}$. In particular, $W$ is conformal. By a result of K. Yano and T. Nagano (see [21]), either $W$ is Killing or $\left(M^{4}, g\right)$ is $S^{4}$ with its canonical metric. But in the latter case $W$ would have two zeroes. The proof follows from Theorem 3.1.

Proposition 3.6. Let $\left(M^{4}, g\right)$ be a compact Einstein manifold $\left({ }^{M_{\text {Ricci }}=}\right.$ $c^{M} g$ ) of dimension four and let $\varphi:\left(M^{4}, g\right) \rightarrow\left(N^{3}, h\right)$ be a nonconstant harmonic morphism with orientable vertical distribution.

Then there exists a Killing field tangent to the fibres of $\varphi$ and nowhere zero on the set of regular points. In particular, the Euler number of $M^{4}$ is equal to the number of critical points of $\varphi$.

If $\varphi$ has critical points, then $c^{M}>0$ and ${ }^{N} b_{1}={ }^{N} b_{2}=0$, where ${ }^{N} b_{1}$ and $\mathrm{N}_{b_{2}}$ denote the Betti numbers of $N^{3}$.

Proof. Because $\varphi$ has compact fibres it cannot be of the third type.

From Corollary 3.3 and Corollary 3.5 it follows that there exists a Killing vector field tangent to the fibres of $\varphi$. Then the fact that $c^{M}>0$ follows from Corollary 3.3 and equation (3.1). Also by a well-known result of S. Bochner we have ${ }^{M} b_{1}=0$. From $[11,7.14]$ it follows that ${ }^{N} b_{1}=0$ and by Poincaré duality ${ }^{N} b_{2}={ }^{N} b_{1}=0$.

Remark 3.7. More generally, if $M^{4}$ is an arbitrary compact manifold then the number of critical points of $\varphi: M^{4} \rightarrow N^{3}$ is equal to the Euler number of $M^{4}$ provided that the smooth map $\varphi$ has isolated critical points (this follows from [33, Proposition 4.2(iii)]).

Recall that by the well-known result of M. Berger the Euler number of $\left(M^{4}, g\right)$ (Einstein and compact) is nonnegative and is zero if and only if $\left(M^{4}, g\right)$ is flat (see $\left.[6,6.32]\right)$.

We can now completely describe submersive harmonic morphisms with one-dimensional fibres defined on a compact Einstein four-manifold as follows. 
Theorem 3.8. Let $\left(M^{4}, g\right)$ be a compact Einstein manifold of dimension four. Let $\varphi:\left(M^{4}, g\right) \rightarrow\left(N^{3}, h\right)$ be a submersive harmonic morphism.

Then $\left(M^{4}, g\right)$ and $\left(N^{3}, h\right)$ are flat and the fibres of $\varphi$ are locally generated by parallel vector fields. In particular, up to homotheties and Riemannian coverings $\varphi$ is the canonical projection between flat tori $T^{4} \rightarrow T^{3}$.

Proof. By passing, to a two-fold covering if necessary we can suppose that the vertical distribution of $\varphi$ is orientable. Then, from Proposition 3.6 it follows that there exists a Killing vector field $V$ on $\left(M^{4}, g\right)$ tangent to the fibres of $\varphi$ and the Euler number of $M^{4}$ is zero. Hence $\left(M^{4}, g\right)$ is flat by the above mentioned result of Berger. From a result of Bochner (see $[6,1.84]$ ) $V$ is parallel.

The fact that $\left(N^{3}, h\right)$ is flat follows from Lemma 1.11.

Because $\varphi$ has compact regular fibres, from a well-known result of C. Ehresmann [13], it follows that the leaf space of the foliation whose leaves are the connected components of the regular fibres of $\varphi$ is smooth. Thus by factorising $\varphi$, if necessary, into a harmonic morphism followed by a Riemannian covering we can suppose that $\varphi$ has connected fibres. Thus, $\varphi$ is, up to homotheties, the quotient induced by $V$. Hence, $\varphi$ is the projection of a $S^{1}$-principal bundle and the horizontal distribution $\mathcal{H}$ is a flat principal connection on it. Then, each holonomy bundle $P$ of it is a regular covering over $N^{3}$ with group the holonomy group $H\left(\subseteq S^{1}\right)$ of $\mathcal{H}$. Moreover, because $\left(M^{4}, g\right)$ is flat, $P$ considered with the metric induced by $g$ is flat (actually, up to homotheties, this is the unique metric with respect to which $P \rightarrow N$ becomes a Riemannian covering; in particular, $P$ with the considered metric is complete.) Hence $M=P \times_{H} S^{1}$ and the pull back of $\varphi$ by $P \rightarrow N$ is the projection $P \times S^{1} \rightarrow P$. To end the proof, recall ([22, Chapter V, Theorem $4.2])$ that $P$ is covered by an Euclidean cylinder or by a torus.

\section{Remark 3.9.}

1) The condition that $\varphi$ is submersive, in Theorem 3.8, can be removed if we assume that $\left(N^{3}, h\right)$ is of constant curvature (see Theorem 4.11).

2) Recall that the K3 surfaces and the tori cannot carry any metric of positive scalar curvature (see [6, 4.34]). Hence, from Proposition 3.6 and Theorem 3.8 we obtain the following:

- There exists no K3 surface endowed with an Einstein metric and which is the domain of a harmonic morphism whose regular fibres are of dimension one. 
- Let $g$ be an Einstein metric on the torus $T^{4}$ and let $\varphi:\left(T^{4}, g\right) \rightarrow$ $\left(N^{3}, h\right)$ be a nonconstant harmonic morphism. Then $g$ and $h$ are flat, $\varphi$ is submersive and, up to homotheties and Riemannian coverings, $\varphi$ is the canonical projection $T^{4} \rightarrow T^{3}$.

Corollary 3.10. Let $\left(M^{4}, g\right)$ be a compact Einstein manifold of dimension four.

Then there exists no submersive harmonic morphism $\varphi:\left(M^{4}, g\right) \rightarrow$ $\left(N^{3}, h\right)$ if either ${ }^{N} b_{1}=0$ or ${ }^{M} b_{1}=0$.

\section{Harmonic morphisms $\varphi:\left(M^{4}, g\right) \rightarrow\left(N^{3}, h\right)$ between Einstein manifolds.}

In this section $\left(M^{4}, g\right)$ and $\left(N^{3}, h\right)$ will be Einstein manifolds (since $N^{3}$ is three-dimensional this means that $\left(N^{3}, h\right)$ is of constant curvature) and $\varphi:\left(M^{4}, g\right) \rightarrow\left(N^{3}, h\right)$ will be a harmonic morphism. Recall that, by a result of P. Baird [2, Proposition 5.1], the set of critical points of $\varphi$ is discrete and hence, by the second axiom of countability, at most countable.

We now state one of the main results of this section enumerating all surjective submersive harmonic morphisms between complete simply-connected Einstein manifolds of dimension four and three, respectively.

Theorem 4.1. Let $\left(M^{4}, g\right)$ be a complete simply-connected Einstein manifold and let $\left(N^{3}, h\right)$ be complete, simply-connected and with constant curvature.

Let $\varphi:\left(M^{4}, g\right) \rightarrow\left(N^{3}, h\right)$ be a surjective submersive harmonic morphism with connected fibres.

Then, up to homotheties, $\varphi$ is one of the following projections $\mathbb{R}^{4} \rightarrow \mathbb{R}^{3}$, $H^{4} \rightarrow \mathbb{R}^{3}, H^{4} \rightarrow H^{3}$ induced by the following canonical warped-product decompositions $\mathbb{R}^{4}=\mathbb{R}^{1} \times \mathbb{R}^{3}, H^{4}=H^{1} \times_{r} \mathbb{R}^{3}, H^{4}=H^{1} \times_{s} H^{3}$ where $H^{k}$ is the hyperbolic space of dimension $k$.

Proof. First we prove that $\left(M^{4}, g\right)$ has constant curvature and that $\varphi$ has geodesic fibres and integrable horizontal distribution.

By Theorem 3.1, either (i) the vertical distribution of $\varphi$ is Riemannian and locally generated by Killing fields or (ii) $\varphi$ has geodesic fibres and integrable horizontal distribution. Suppose that case (i) holds and let $\mathcal{V}=\operatorname{ker} \varphi_{*}$ be the vertical distribution. We can choose a local orthonormal frame $\{X, Y, Z\}$ for $\mathcal{H}\left(=\mathcal{V}^{\perp}\right)$ with respect to $h$ made up of basic fields and such that $i_{Z} \Omega=0$. 
Because $\left(M^{4}, g\right)$ and $\left(N^{3}, h\right)$ are both Einstein, from (1.1) we obtain

$$
X(\sigma) Y(\sigma)=0 \quad \text { and } \quad X(\sigma)^{2}=Y(\sigma)^{2} .
$$

Thus $X(\sigma)=Y(\sigma)=0$.

From (1.1) it follows that (1.14) holds after, replacing, if necessary, one of the vector fields $X, Y, Z$ or $V$ with its negative. Choose the (local) orientation on $N^{3}$ such that $\left\{\varphi_{*} X, \varphi_{*} Y, \varphi_{*} Z\right\}$ is positively oriented.

Let $\check{\lambda}$ be the function on $N^{3}$ such that $e^{\sigma}=\varphi^{*}(\check{\lambda})$ and let $F$ be the two-form on $N^{3}$ such that $\Omega=\varphi^{*}(F)$. Then (1.14) implies that

$$
F=* \mathrm{~d} \check{\lambda}^{-2} \text {. }
$$

where $*$ is the Hodge star-operator on $\left(N^{3}, h\right)$.

But $\mathrm{d} F=0$ and thus (4.2) implies that $\check{\lambda}^{-2}$ is a positive harmonic function on $\left(N^{3}, h\right)$.

From (1.1) and (1.3) we obtain

$$
k^{N}=\check{\lambda}^{-2} c^{M}
$$

where $k^{N}$ is the constant sectional curvature of $\left(N^{3}, h\right)$ and $c^{M}$ is the Einstein constant of $\left(M^{4}, g\right)$. Thus either $\check{\lambda}$ is constant or $k^{N}=c^{M}=0$. But in the latter case, by Liouville's theorem, $\check{\lambda}^{-2}$ must be constant. Hence $\check{\lambda}$ is constant and, by [3, Theorem 5.2], $\varphi$ has geodesic fibres. Moreover, by (4.2), $F=0$ and thus $\mathcal{H}$ is integrable.

Thus, we always have case (ii). The fact that $\left(M^{4}, g\right)$ has constant curvature now follows from Corollary A.3.

By [5] $\mathcal{H}$ is an Ehresmann connection for $\varphi$.

Because $\mathcal{H}$ is a flat (i.e., integrable) Ehresmann connection any maximal integral submanifold of it is a covering space of $N^{3}$. But $N^{3}$ is simplyconnected and hence $\varphi$ admits a (global) horizontal section. The proof of the theorem follows.

Corollary 4.2. Let $\left(M^{4}, g\right)$ be a complete simply-connected Einstein manifold and let $\left(N^{3}, h\right)$ be complete, simply-connected and with constant curvature.

Let $\varphi:\left(M^{4}, g\right) \rightarrow\left(N^{3}, h\right)$ be a surjective submersive harmonic morphism with connected fibres; denote its dilation by $\lambda$. Let $W$ be the vertical vector field such that $g(W, W)=\lambda^{-2}$. Suppose that $W$ is complete.

Then, up to homotheties, $\varphi$ is the orthogonal projection $\mathbb{R}^{4} \rightarrow \mathbb{R}^{3}$. 
Proof. This follows from Corollary 3.5 and Theorem 4.1.

Proposition 4.3. Let $\varphi:\left(M^{4}, g\right) \rightarrow\left(N^{3}, h\right)$ be a harmonic morphism between Einstein manifolds and let $\lambda$ be its dilation. Suppose that the regular fibres of $\varphi$ form a Riemannian foliation.

Then, up to homotheties, $\varphi$ can be (locally) characterised as follows:

- $\left(M^{4}, g\right)$ is Ricci-flat and $\left(N^{3}, h\right)$ is flat;

- $\lambda^{-2}$ is the pull back of a local positive harmonic function $u$ on $\left(N^{3}, h\right)$ (in particular, $\lambda^{-2}$ is a harmonic function on $\left(M^{4}, g\right)$ );

- Any local connection form $A\left(=s^{*} \theta\right)$ of the horizontal distribution satisfies

$$
\mathrm{d} A=* \mathrm{~d} u
$$

where $*$ is the Hodge star-operator of $\left(N^{3}, h\right)$ with respect to some (local) orientation (equivalently, the curvature form $F=\mathrm{d} A$ satisfies the monopole equation $F=* \mathrm{~d} u$ );

- In a neighbourhood of the local section $s$ of $\varphi$ where $\varphi$ is equivalent to a projection we have

$$
g=u h+u^{-1}(\mathrm{~d} t+A)^{2} .
$$

Proof. This follows from the proof of Theorem 4.1.

\section{Remark 4.4.}

1) Note that the metric $g$ of Proposition 4.3 is constructed by applying S.W. Hawking's ansatz [19] (cf. [25]).

2) Let $\varphi:\left(M^{4}, g\right) \rightarrow\left(N^{3}, h\right)$ be a harmonic morphism between Einstein manifolds. If $\left(M^{4}, g\right)$ does not have constant curvature or the horizontal distribution is nonintegrable then $\left(M^{4}, g\right)$ is Ricci-flat and $\varphi$ is of type (i) (and hence locally given as in Proposition 4.3) or type (iii) of Theorem 1.8. This follows from Theorem 1.8 and Corollary A.3.

Let $a \geq 0$. Recall that if we apply the Hawking's ansatz (with the convention $\mathrm{d} A=-* \mathrm{~d} u)$ to the harmonic function $u_{a}: \mathbb{R}^{3} \backslash\{0\} \rightarrow(0, \infty)$ defined by $u_{a}(y)=\frac{1}{4}\left(\frac{1}{|y|}+a\right), y \in \mathbb{R}^{3} \backslash\{0\}$, then the following metric is obtained. 
Definition 4.5. Let $a>0$. The Hawking Taub-NUT metric is the Riemannian metric on $\mathbb{R}^{4}$ defined by

$$
g_{a}=\left(a|x|^{2}+1\right) g_{0}-\frac{a\left(a|x|^{2}+2\right)}{a|x|^{2}+1}\left(-x^{2} \mathrm{~d} x^{1}+x^{1} \mathrm{~d} x^{2}-x^{4} \mathrm{~d} x^{3}+x^{3} \mathrm{~d} x^{4}\right)^{2} .
$$

For $a=0$ this gives the canonical metric $g_{0}$ on $\mathbb{R}^{4}$.

Note that $g_{1}$ is discussed in [25].

\section{Remark 4.6.}

1) For any $a \geq 0$ the Hopf polynomial $\varphi:\left(\mathbb{R}^{4}, g_{a}\right) \rightarrow\left(\mathbb{R}^{3}, h_{0}\right)$ is the harmonic morphism induced by the isometric action of $S^{1}$ on $\left(\mathbb{R}^{4}, g_{a}\right)$ where $h_{0}$ is the canonical metric on $\mathbb{R}^{3}$. In particular, $\left(\mathbb{R}^{4}, g_{a}\right)$ is Ricciflat for any $a \geq 0$.

2) Moreover, we can consider $a=\varphi^{*}(\check{a})$ to be the pull back of a nonnegative harmonic function $\check{a}$ defined in the neighbourhood of $0 \in \mathbb{R}^{3}$. Then, the resulting metric $g_{a}$ is still Ricci-flat and with respect to it the Hopf polynomial, suitably restricted, is a harmonic morphism.

For the next construction we follow C. LeBrun's discussion [25] of Hawking's ansatz [19].

Example 4.7. Let $u: \mathbb{R}^{3} \backslash C_{u} \rightarrow(0, \infty)$ be a positive harmonic function whose set of singularities $C_{u}=\left\{y_{j}\right\}_{j \in I}$ is discrete. Hence $I$ is finite or countable. Thus by applying Bôcher's theorem, the 'minimum' and Harnack's principles (see [1]) we obtain

$$
u(y)=a+\sum_{j \in I} \frac{b_{j}}{\left|y-y_{j}\right|}
$$

for any $y \in \mathbb{R}^{3}$ where $a \geq 0$ and $b_{j} \geq 0$ are nonnegative constants. Suppose that $u$ has the same residue equal to $b(>0)$ at each singular point, i.e., $b_{j}=b$ for each $j \in I$.

Let $F_{u} \in \Gamma\left(\Lambda^{2}\left(T^{*}\left(\mathbb{R}^{3} \backslash C_{u}\right)\right)\right)$ be defined by $F_{u}=-* \mathrm{~d} u$ where $*$ is the Hodge star-operator on $\mathbb{R}^{3}$. Because $u$ is harmonic we have $\mathrm{d} F_{u}=0$. Then, taking $S^{1}=\mathbb{R} / 4 \pi b \mathbb{Z}$, the cohomology class

$$
\frac{1}{4 \pi b}\left[F_{u}\right] \in H^{2}\left(\mathbb{R}^{3} \backslash C_{u}, \mathbb{Z}\right)=H^{1}\left(\mathbb{R}^{3} \backslash C_{u}, S^{1}\right)
$$


is the first Chern class of a principal bundle $\xi_{u}=\left(P_{u}, \mathbb{R}^{3} \backslash C_{u}, S^{1}\right)$, with projection $\psi_{u}: P_{u} \rightarrow \mathbb{R}^{3} \backslash C_{u}$. It is not difficult to see, by using the homotopy sequence of $\xi_{u}$, that $P_{u}$ is simply-connected.

As is well-known, $F_{u}$ is the curvature form of a principal connection given by $\theta_{u} \in \Gamma\left(T^{*} P_{u}\right)$. Note that if $A$ is a local connection form of $\theta_{u}$, with respect to some local section of $\xi_{u}$, then $\mathrm{d} A=-* \mathrm{~d} u$.

Let $h_{0}$ be the canonical metric on $\mathbb{R}^{3}$ and define $\gamma_{u}=\psi_{u}^{*}\left(u h_{0}\right)+$ $\psi_{u}^{*}\left(u^{-1}\right) \theta_{u}^{2}$. Then $\psi_{u}:\left(P_{u}, \gamma_{u}\right) \rightarrow\left(\mathbb{R}^{3} \backslash C_{u},\left.h_{0}\right|_{\mathbb{R}^{3} \backslash C_{u}}\right)$ is a harmonic morphism.

The key point of the construction is the fact that $\psi_{u}$ can be extended to a harmonic morphism whose codomain is $\mathbb{R}^{3}$.

To prove this, first note that if $C_{u}=\{0\}$ then $\xi_{u}$ is the cylinder of the Hopf bundle $\left(S^{3}, S^{2}, S^{1}\right)$ and hence $\psi_{u}$ is the restriction of the Hopf polynomial to $\mathbb{R}^{4} \backslash\{0\}$. Moreover, one can easily verify that $\gamma_{u}$ is homothetic to the restriction of the Hawking Taub-NUT metric $g_{4 a}$ to $\mathbb{R}^{4} \backslash\{0\}$ where, from now on, we consider, for simplicity, that $b=\frac{1}{4}$.

Let $v(y)=\frac{b}{\left|y-y_{1}\right|}$ and $w=u-v$. Then

$$
\xi_{u}=\xi_{v+w}=\left.\left.\xi_{v}\right|_{\mathbb{R}^{3} \backslash C_{u}} \cdot \xi_{w}\right|_{\mathbb{R}^{3} \backslash C_{u}}
$$

where '.' denotes the group operation in $H^{1}\left(\mathbb{R}^{3} \backslash C_{u}, S^{1}\right)$. There exists a neighbourhood $U$ of $y_{1}$ such that $U \cap C_{u}=\left\{y_{1}\right\}$ and hence $\left.w\right|_{U}$ is a (welldefined) positive harmonic function. By taking $U$ to be contractible we get that $\left.\xi_{w}\right|_{U}$ is trivial (equivalently, it is the neutral element of $H^{1}\left(U, S^{1}\right)$ ). Then $\left.\xi_{u}\right|_{U \backslash\left\{y_{1}\right\}}=\left.\xi_{v}\right|_{U \backslash\left\{y_{1}\right\}}$ and hence $\psi_{u}$ can be extended so that its image contains $y_{1}$. More precisely, we can add a point $x_{1}$ to $\psi_{u}^{-1}(U)$ such that the extended map is smoothly equivalent, in a neighbourhood of $x_{1}$, to the cone of the Hopf fibration $S^{3} \rightarrow S^{2}$. Moreover, because $w$ has no singularities in $U$ the metric $\gamma_{u}$ extends over $x_{1}$ to a metric which is homothetic, in the neighbourhood of $x_{1}$, to $g_{4 w}$ of Remark 4.6(2).

In this way $\left(P_{u}, \gamma_{u}\right)$ can be extended to a Riemannian manifold $\left(M_{u}, g_{u}\right)$ and $\psi_{u}$ can be extended to a surjective harmonic morphism $\varphi_{u}:\left(M_{u}, g_{u}\right) \rightarrow\left(\mathbb{R}^{3}, h_{0}\right)$ where $h_{0}$ is the canonical metric on $\mathbb{R}^{3}$. Note that $\left(M_{u}, g_{u}\right)$ is Ricci-flat, simply-connected and that $\varphi_{u}$ is induced by an isometric action.

We can now state the next main result of this section enumerating all the surjective harmonic morphisms with critical points between complete, simply-connected Einstein manifolds of dimension four and three, respectively. 
Theorem 4.8. Let $\left(M^{4}, g\right)$ be a complete Einstein manifold and let $\left(N^{3}, h\right)$ be complete, simply-connected and with constant curvature.

Let $\varphi:\left(M^{4}, g\right) \rightarrow\left(N^{3}, h\right)$ be a surjective harmonic morphism with orientable vertical distribution; denote its dilation by $\lambda$. Suppose that $\varphi$ has critical points.

Then, up to homotheties, $\left(N^{3}, h\right)=\left(\mathbb{R}^{3}, h_{0}\right)$ where $h_{0}$ is the canonical metric on $\mathbb{R}^{3}$. Moreover, $\lambda^{-2}=\varphi^{*}(u)$ for a positive harmonic function $u: \mathbb{R}^{3} \backslash C_{u} \rightarrow(0, \infty)$ having the same (positive) residue at each (fundamental) pole $y \in C_{u}$ and $\left(M^{4}, g\right)=\left(M_{u}^{4}, g_{u}\right)$ and $\varphi=\varphi_{u}$.

Proof. By Corollary 3.3 there exists a Killing field $V$ on $(M, g)$ tangent to the fibres of $\varphi$.

Although $\varphi$ has critical points, an argument due to R. Hermann (see [6, 9.45]) can be adapted to prove that the horizontal distribution $\mathcal{H}$ (which is well-defined outside the set of critical points) is an Ehresmann connection for $\varphi$ restricted to the set of regular points. By applying [6, 9.40], it is easy to see that $\varphi$ can be factorised into a harmonic morphism with connected fibres followed by a Riemannian covering over $\left(N^{3}, h\right)$. But the latter must be trivial because $N^{3}$ is simply-connected and hence $\varphi$ has connected fibres.

Now, as in the proof of Theorem 4.1 we obtain (4.3) and the monopole equation (4.2) and hence $\check{\lambda}^{-2}$ is a harmonic function where $\lambda=\varphi^{*}(\check{\lambda})$.

Because $\varphi$ has critical points its dilation cannot be constant. This, together with (4.3), imply that $\left(M^{4}, g\right)$ is Ricci-flat and $\left(N^{3}, h\right)$ is flat. Hence, up to homotheties, $\left(N^{3}, h\right)=\left(\mathbb{R}^{3}, h_{0}\right)$ where $h_{0}$ is the canonical metric on $\mathbb{R}^{3}$.

Using the completeness of $\left(M^{4}, g\right)$ and the fact that $V$ is Killing it is not difficult to prove (directly or by using [29, Theorem 2.9]) that the restriction of $\varphi$ to the set of regular points is the projection of a principal bundle $\xi$ with group $(\mathbb{R},+)$ or $\left(S^{1}, \cdot\right)$ and the horizontal distribution is a principal connection on it. But $\varphi$ extends the projection of $\xi$ over the critical points. Hence in the neighbourhood of each critical point $\xi$ is (suitably restricted) the cylinder of the Hopf bundle $\left(S^{3}, S^{2}, S^{1}\right)$ or its dual. Hence the structural group of $\xi$ is $S^{1}=\mathbb{R} / L \mathbb{Z}$ where $L(>0)$ is the period of the orbits of $V$.

Let $\left\{y_{j}\right\}_{j \in I}$ be the set of critical values of $\varphi$. Using the Chern-Weil morphism and (4.2) it is easy to see that the first Chern number of $\xi$ suitably restricted to a sphere about any $y_{j}$ is given by $c_{1}=-4 \pi b_{j} / L$ where $b_{j}(>0)$ is the residue of $\check{\lambda}^{-2}$ at $y_{j}$. But we must have $c_{1}= \pm 1$ and hence $b_{j}=b_{k}$ for any $j, k \in I$ and the proof follows. 
Remark 4.9. Note that the period $L$ of $V$ is the mass of the regular fibres, i.e., $L=\int_{\text {fibre }} \rho$ where $\rho\left(=\lambda^{-1}\right)$ is the density of $\varphi$. Because $\rho$ is constant along the fibres we have that $L$ is equal to $\left.\rho\right|_{\text {fibre }}$ multiplied by the length of the considered fibre.

We end with two other classification results:

Theorem 4.10. Let $\left(M^{4}, g\right)$ be a complete simply-connected Einstein manifold and let $\left(N^{3}, h\right)$ be complete, simply-connected and with constant curvature.

Let $\varphi:\left(M^{4}, g\right) \rightarrow\left(N^{3}, h\right)$ be a surjective harmonic morphism. Suppose that $\varphi$ has exactly one critical point.

Then there exists $a \geq 0$ such that, up to homotheties, $\varphi:\left(\mathbb{R}^{4}, g_{a}\right) \rightarrow$ $\left(\mathbb{R}^{3}, h_{0}\right)$ is the Hopf polynomial with $g_{a}$ the Hawking Taub-NUT metric $(a>$ $0)$ and $g_{0}, h_{0}$ the canonical metrics on $\mathbb{R}^{4}, \mathbb{R}^{3}$, respectively.

Proof. This follows from Theorem 4.8.

Theorem 4.11. Let $\left(M^{4}, g\right)$ be a compact Einstein manifolds of dimension four. Let $\left(N^{3}, h\right)$ be a Riemannian manifold of dimension three with constant curvature. Let $\varphi:\left(M^{4}, g\right) \rightarrow\left(N^{3}, h\right)$ be a nonconstant harmonic morphism.

Then $\left(M^{4}, g\right)$ and $\left(N^{3}, h\right)$ are flat, $\varphi$ is submersive and its fibres are locally generated by parallel vector fields. In particular, up to homotheties and Riemannian coverings $\varphi$ is the canonical projection $T^{4} \rightarrow T^{3}$ between flat tori.

In particular there exists no harmonic morphism with one-dimensional fibres from a compact Einstein manifold of dimension four to $S^{3}$.

Proof. Suppose that $\varphi$ has critical points. Then by Proposition 3.6 we have that $c^{M}>0$. From (4.3) it follows that the dilation of $\varphi$ is constant and hence $\varphi$ cannot have critical points.

Hence $\varphi$ is submersive and the proof follows from Theorem 3.8.

Remark 4.12. We do not know any example of a harmonic morphism with critical points and one-dimensional regular fibres which is defined on a compact four-dimensional Einstein manifold. 


\section{A. Appendix. \\ Some constructions of one-dimensional foliations which produce harmonic morphisms on Einstein manifolds.}

Here we use well-known results on warped-products and conformal vector fields (see [6, Chapter 9, Section J], [23]) to show that there exist onedimensional foliations with integrable orthogonal complement and which produce harmonic morphisms on Einstein manifolds which are not of constant curvature (thus answering to a question formulated by S. Gudmundsson). Also, we give a result needed in the proof of Theorem 4.1.

\section{A.1. Homothetic foliations with geodesic leaves.}

Proposition A.1 (cf. [6, Chapter 9, Section J]). Let $\varphi:\left(M^{n+1}, g\right) \rightarrow$ $\left(N^{n}, h\right), n \geq 3$, be a nonconstant harmonic morphism with geodesic leaves and integrable horizontal distribution; denote its dilation by $\lambda$. Then the following assertions are equivalent.

(i) $\left(M^{n+1}, g\right)$ is Einstein $\left({ }^{M}\right.$ Ricci $\left.=c^{M} g\right)$;

(ii) $\left(N^{n}, h\right)$ is Einstein $\left({ }^{N}\right.$ Ricci $\left.=c^{N} h\right)$ and the following relation holds

$$
\frac{c^{M}}{n} \lambda^{2}-\frac{c^{N}}{n-1} \lambda^{4}+(U(\lambda))^{2}=0
$$

where $U$ is a vertical vector field such that $g(U, U)=1$.

Moreover, if (i) or (ii) holds then

$$
K_{X \wedge Y}^{M}-\frac{c^{M}}{n}=\lambda^{2}\left(K_{\varphi_{*} X \wedge \varphi_{*} Y}^{N}-\frac{c^{N}}{n-1}\right)
$$

where $K^{M}$ and $K^{N}$ are the sectional curvature of $(M, g)$ and $(N, h)$, respectively, and $X, Y$ are horizontal.

Proof. The equivalence (i) $\Longleftrightarrow$ (ii) follows from Lemma 4.6, (4.4), (5.3) and (5.4) of [29] or from [6,9.107-109] (note that (A.1) is essentially the same as equation (9.109) of [6]).

If (i) or (ii) holds then (A.2) follows from (A.1) and the following formula

$$
\lambda^{2} K_{X \wedge Y}^{M}-\lambda^{4} K_{\varphi_{*} X \wedge \varphi_{*} Y}^{N}+(U(\lambda))^{2}=0
$$

which can be obtained directly or as a consequence of a formula of S. Gudmundsson [17]. 
Proposition A.2 (cf. [23, Lemma 13(iv)]). Let $\varphi:\left(M^{n+1}, g\right) \rightarrow\left(N^{n}\right.$, $h), n \geq 3$, be a nonconstant harmonic morphism with geodesic leaves and integrable horizontal distribution.

(i) If $\left(M^{n+1}, g\right)$ has constant curvature then $\left(N^{n}, h\right)$ has constant curvature.

(ii) If $\left(M^{n+1}, g\right)$ is Einstein and $\left(N^{n}, h\right)$ has constant curvature then $\left(M^{n+1}, g\right)$ has constant curvature.

Proof. Assertion (i) is an immediate consequence of (A.2).

If $\left(M^{n+1}, g\right)$ is Einstein then from (A.1) and from (5.1) of [29] we obtain

$$
K_{X \wedge U}^{M}=\frac{c^{M}}{n}
$$

where $X$ is any horizontal vector.

The proof of (ii) follows from (A.2) and (A.3).

The following corollary is needed in the proof of Theorem 4.1.

Corollary A.3 (cf. [23, Corollary 15]). Let $\varphi:\left(M^{4}, g\right) \rightarrow\left(N^{3}, h\right)$ be a harmonic morphism with one-dimensional geodesic leaves and integrable horizontal distribution.

If $\left(M^{4}, g\right)$ is Einstein then both $\left(M^{4}, g\right)$ and $\left(N^{3}, h\right)$ have constant curvature.

Proof. If $\left(M^{4}, g\right)$ is Einstein then by Proposition A.1, $\left(N^{3}, h\right)$ is Einstein. But $N^{3}$ is three-dimensional and thus $\left(N^{3}, h\right)$ has constant curvature. The proof follows from Proposition A.2(ii).

Corollary A.4 (cf. [23], [6, Chapter 9, Section J]). Given any Einstein manifold $\left(N^{n}, h\right)$ of dimension $n$ there exists an Einstein manifold $\left(M^{n+1}, g\right)$ of dimension $n+1$ and a harmonic morphism $\varphi:\left(M^{n+1}, g\right) \rightarrow$ $\left(N^{n}, h\right)$ with geodesic fibres and integrable horizontal distribution.

If $n \geq 4$ and $\left(N^{n}, h\right)$ does not have constant curvature then $\left(M^{n+1}, g\right)$ does not have constant curvature.

Proof. Let $\left(N^{n}, h\right)$ be Einstein and let $\lambda$ be a (local) solution of (A.1) (see $[6,9.109])$. 
Let $M^{n+1}=\mathbb{R} \times N^{n}$ and $g=\mathrm{d} t^{2}+\lambda^{-2} h$. It is obvious that the canonical projection $\left(M^{n+1}, g\right) \rightarrow\left(N^{n}, h\right)$ is a harmonic morphism with geodesic leaves and integrable horizontal distribution. Also $\left(M^{n+1}, g\right)$ is an Einstein manifold by Proposition A.1. Moreover, if $\left(N^{n}, h\right)$ does not have constant curvature then, by Proposition A.2, $\left(M^{n+1}, g\right)$ does not have constant curvature.

\section{A.2. Riemannian foliations locally generated by Killing fields.}

The following results are consequences of [6, Corollary 9.107, 9.108, 9.109].

Proposition A.5 ([6]). Let $\left(N^{n}, h\right)$ be a Riemannian manifold and $\rho$ : $N^{n} \rightarrow(0, \infty)$ a smooth positive function.

Let $M^{n+1}=\mathbb{R} \times N^{n}$ and $g=\rho^{2} \mathrm{~d} t^{2}+h$. Then, the following assertions are equivalent.

(i) $\left(M^{n+1}, g\right)$ is Einstein $\left({ }^{M}\right.$ Ricci $\left.=c^{M} g\right)$.

(ii) $\left(N^{n}, h\right)$ has constant scalar curvature $s^{N}=(n-1) c^{M}$ and the following relation holds

$$
\stackrel{N}{\nabla} \mathrm{d} \rho=-\frac{c^{M}}{n} \rho h+\rho Z^{N}
$$

where $\stackrel{N}{\nabla}$ is the Levi-Civita connection on $\left(N^{n}, h\right)$ and $Z^{N}={ }^{N}$ Ricci $\left(s^{N} / n\right) h$ is the trace-free part of ${ }^{N}$ Ricci .

Proof. From [6, 9.106a, 9.106c] or, by a straightforward calculation the following equations can be obtained:

$$
\begin{aligned}
p_{N}^{*}\left({ }^{M} \text { Ricci }\right) & ={ }^{N} \text { Ricci }-\rho^{-1} \nabla^{N} \mathrm{~d} \rho \\
p_{\mathbb{R}}^{*}\left({ }^{M} \text { Ricci }\right) & =\rho^{-1}\left(\Delta^{N} \rho\right) p_{\mathbb{R}}^{*}(g)
\end{aligned}
$$

where $p_{N}: M \rightarrow N$ and $p_{\mathbb{R}}: M \rightarrow \mathbb{R}$ are the canonical projections. Also, $[6,9.106 \mathrm{~b}]$ gives that ${ }^{M} \operatorname{Ricci}(X, \partial / \partial t)=0$ for any $X \in \Gamma(T N)$. From this and (A.5) the proof easily follows.

Corollary A.6. For each $n \geq 5$ there exists Einstein manifolds $\left(M^{n+1}, g\right)$ not of constant curvature, endowed with a nowhere zero Killing field which has integrable orthogonal complement. Moreover, the construction can be done in such a way that the (locally) induced isometric quotients are also Einstein. 
Proof. If the equation $\stackrel{N}{\nabla} \mathrm{d} \rho=a \rho h$, where $a \in \mathbb{R}$, has solutions then there exists a homothetic one-dimensional foliation with geodesic leaves and integrable orthogonal complement (see [30]). Recall that $Z^{N}=0$ if and only if $\left(N^{n}, h\right)$ is Einstein (see $\left.[6,1.118]\right)$. Hence, $[6,9.109]$ and Corollary A.4 implies that there exists an Einstein manifold $\left(N^{n}, h\right), n \geq 5$, not of constant curvature, on which (A.4) has a (local) solution $\rho$ which is positive. Then, by Proposition A.5, $\left(M^{n+1}, g\right)$ (where $M^{n+1}=\mathbb{R} \times N^{n}$ and $g=\rho^{2} \mathrm{~d} t^{2}+h$ ) is Einstein. Clearly $V=\partial / \partial t$ is a nowhere zero Killing field on $\left(M^{n+1}, g\right)$.

\section{References.}

[1] S. Axler, P. Bourdon, and W. Ramey, Harmonic function theory, Graduate Texts in Mathematics, 137, Springer-Verlag, New-York, 1992.

[2] P. Baird, Harmonic morphisms and circle actions on 3- and 4- manifolds, Ann. Inst. Fourier (Grenoble), 40(1) (1990), 177-212.

[3] P. Baird and J. Eells, A conservation law for harmonic maps, Geometry Symposium. Utrecht 1980, 1-25, Lecture Notes in Math., 894, SpringerVerlag, Berlin, Heidelberg, New York, 1981.

[4] P. Baird and J.C. Wood, Harmonic morphisms, Seifert fibre spaces and conformal foliations, Proc. London Math. Soc., 64 (1992), 170-196.

[5] P. Baird and J.C. Wood, Harmonic morphisms between Riemannian manifolds, book in preparation, London Math. Soc. Monogr. (N.S.), Oxford Univ. Press.

[6] A.L. Besse, Einstein manifolds, Ergebnisse der Mathematik und ihrer Grenzgebiete (3), 10, Springer-Verlag, Berlin-New York, 1987.

[7] R.L. Bryant, Harmonic morphisms with fibres of dimension one, Comm. Anal. Geom., 8 (2000), 219-265.

[8] F. Burstall, D. Ferrus, K. Leshke, F. Pedit, and U. Pinkall, Conformal Geometry of Surfaces in the 4-Sphere and Quaternions, Preprint, arXiv:math.DG/0002075, 2000.

[9] D.M.J. Calderbank, Selfdual Einstein metrics and conformal submersions, Preprint, arXiv:math.DG/0001041, 2000. 
[10] P.T. Church and J.G. Timourian, Maps with 0-dimensional critical set, Pacific J. Math., 57(1) (1975), 59-66.

[11] J. Eells and L. Lemaire, Selected topics in harmonic maps, CBMS Regional Conference Series in Mathematics, 50, published for the Conference Board of the Mathematical Sciences, Washington, DC by the American Mathematical Society, Providence, RI, 1983.

[12] T. Eguchi and A.J. Hanson, Asimptotically flat self-dual solutions to Euclidean gravity, Phis. Lett. B, 74 (1978), 249-251.

[13] C. Ehresmann, Les connections infinitésimales dans un espace fibré différentianble, Coloque de Topologie, Bruxelles 1950, Georges Thone, Liège; Masson et Cie., Paris, (1951), 29-55.

[14] B. Fuglede, Harmonic morphisms between Riemannian manifolds, Ann. Inst. Fourier (Grenoble), 28 (1978), 107-144.

[15] B. Fuglede, A criterion of non-vanishing differential of a smooth map, Bull. London Math. Soc., 14 (1982), 98-102.

[16] S. Gallot and D. Meyer, Opérateur de courbure et laplacien des formes différentielles d'une variété riemannienne, J. Math. Pures Appl. (9), 54(3) (1975), 259-284.

[17] S. Gudmundsson, The Geometry of Harmonic Morphisms, Ph.D. thesis, University of Leeds, 1992.

[18] S. Gudmundsson, The Bibliography of Harmonic Morphisms, http:// www.maths.Ith.se/matematiklu/personal/sigma/harmonic/ bibliography.html

[19] S.W. Hawking, Gravitational Instantons, Phys. Lett., 60A (1977), 8183.

[20] T. Ishihara, A mapping of Riemannian manifolds which preserves harmonic functions, J. Math. Kyoto Univ., 19 (1979), 215-229.

[21] S. Kobayashi, Transformation groups in differential geometry, reprint of the 1972 edition, Classics in Mathematics, Springer-Verlag, Berlin, 1995.

[22] S. Kobayashi and K. Nomizu, Foundations of differential geometry, I, II, Interscience Tracts in Pure and Applied Math., 15, Interscience Publ., New York, London, Sydney, 1963, 1969. 
[23] W. Kühnel, Conformal transformations between Einstein spaces, in 'Conformal geometry. Papers from the seminar held in Bonn, 19851986,' R.S. Kulkarni, U. Pinkall (editors), Aspects of Mathematics, E12, Friedr. Vieweg \& Sohn, Braunschweig, (1988), 105-146.

[24] H.B. Lawson, Jr. and M.L. Michelsohn, Spin geometry, Princeton Mathematical Series, 38, Princeton University Press, Princeton, NJ, 1989.

[25] C. LeBrun, Complete Ricci-flat Kähler metrics on $\mathbb{C}^{n}$ need not be flat, in 'Several complex variables and complex geometry,' Part 2 (Santa Cruz, CA, 1989), 297-304, Proc. Sympos. Pure Math., 52, Part 2, Amer. Math. Soc., Providence, RI, 1991.

[26] A. Lichnerowicz, Geometry of groups of transformations, Translated from French and edited by Michael Cole, Noordhoff International Publishing, Leyden, 1977.

[27] X. Mo, Harmonic morphisms via deformation of metrics for horizontally conformal maps, Harmonic morphisms, harmonic maps and related topics, Brest 1997, C.K. Anand, P. Baird, E. Loubeau and J.C. Wood (editors), Pitman Research Notes in Mathematics, CRC Press, (1999), 13-21.

[28] R. Pantilie, On submersive harmonic morphisms, in 'Harmonic morphisms, harmonic maps and related topics,' Brest 1997, C.K. Anand, P. Baird, E. Loubeau and J.C. Wood (editors), Pitman Research Notes in Mathematics, CRC Press, (1999), 23-29.

[29] R. Pantilie, Harmonic morphisms with one-dimensional fibres, Internat. J. Math., 10 (1999), 457-501.

[30] R. Pantilie, Conformal actions and harmonic morphisms, Math. Proc. Cambridge Philos. Soc., 129 (2000), 527-547.

[31] B.L. Reinhart, Differential geometry of foliations. The fundamental integrability problem, Ergebnisse der Mathematik und Ihrer Grenzgebiete, 99, Springer-Verlag, Berlin-New York, 1983.

[32] N.E. Steenrod, The topology of fibre bundles, Princeton Mathematical Series 14, Princeton, Princeton University Press, 1951.

[33] J.G. Timourian, Fibre bundles with discrete singular set, J. Math. Mech., 18 (1968), 61-70. 
[34] J.C. Wood, Harmonic morphisms, foliations and Gauss maps, in 'Complex differential geometry and non-linear differential equations,' 145183, Contemp. Math., 49, Amer Math. Soc., Providence, RI, 1986.

[35] J.C. Wood, Harmonic morphisms and Hermitian structures on Einstein 4-manifolds, Internat. J. Math., 3 (1992), 415-439.

UNIVERSITY OF LEEDS

Dept. of Pure Mathematics

LEEDS LS2 9JT, UK

E-mail address: pmtrp@amsta.leeds.ac.uk

ReCeived March 29, 2000. 